\title{
Analysis of optical back propagation under non-ideal optical phase conjugation and amplifier noise
}

\author{
Guanhui Wang
}

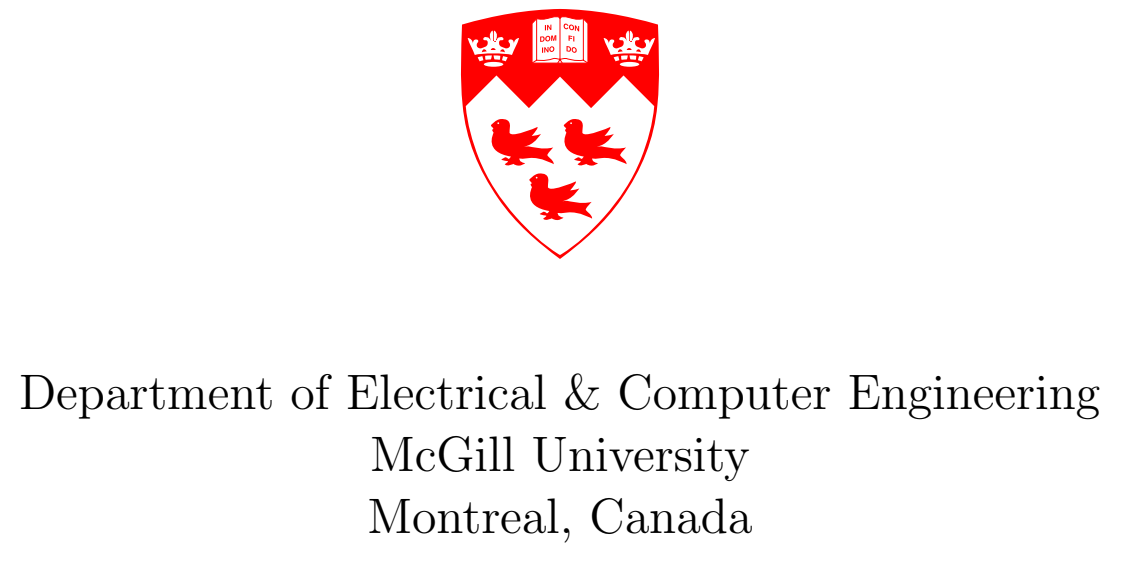

August 2014

A thesis submitted to McGill University in partial fulfillment of the requirements of the degree of Master of Engineering.

(C) 2014 Guanhui Wang 


\begin{abstract}
The continuous growth in the demand of transmission capacity requires high data rates in today's fiber-optic communication systems. Fiber nonlinearity is considered to be the major obstacle in the development of communication capacity. Optical back propagation is one of the ways to address this problem. This thesis explores the performances of optical back propagation under several practical non-ideal conditions. It compares the results of optical back propagation with other nonlinearity control techniques such as digital back propagation and mid-link optical phase conjugation.

Optical back propagation is an all optical process which builds into the optical communication systems to compensate for the phase noise caused by fiber nonlinear effects. Aside from previously established simulation results in the literature of optical back propagation under ideal cases, this thesis demonstrates simulation results of optical back propagation under several non-ideal cases. It demonstrates a $5.7 \mathrm{~dB}$ of power penalty that occurs with a $5 d B$ of optical signal to noise ratio degradation in the system.

For further investigation into the optical back propagation system, advanced modulation formats of the optical signals and higher transmission baud rate effects are studied.

The results demonstrated in this thesis can also be used to determine the optimum operating system parameters under non-ideal conditions in optical back propagation systems.
\end{abstract}




\section{Sommaire}

La croissance incessante de la demande pour la capacité de transmission crée un besoin pour des taux de transmission de données élevés dans les systèmes de communication par fibres optiques d'aujourd'hui. La non-linéarité de la fibre est considérée comme un obstacle majeur dans le développement de la capacité de communication. La rétro-propagation optique est une des façons de répondre à ce problème. Cette thèse explore les performances de la rétro-propagation optique dans plusieurs conditions pratiques et imparfaites. Les résultats obtenus avec la rétro-propagation optique sont comparés à ceux obtenus avec d'autres techniques de contrôle de la non-linéarité telles que la rétro-propagation numérique et la conjugaison de phase optique à mi-chemin.

La rétro-propagation optique est un procédé entièrement optique qui s’introduit dans les systèmes de communication optiques pour compenser le bruit de phase causé par les effets non-linéaires de la fibre. En plus des résultats de simulation institués précédemment dans la documentation pour la rétro-propagation dans des conditions idéales, les résultats de simulation pour la rétro-propagation dans des conditions imparfaites sont présentés dans cette thèse. Il est démontré qu'une perte de puissance de $5.7 d B$ est obtenue lorsque la dégradation du rapport signal sur bruit optique dans le système est de $5 d B$.

Pour de plus amples investigations du système de rétro-propagation optique, des formats de modulation avancés pour le signal optique ainsi que l'effet de l'augmentation de la vitesse de transmission en bauds sont étudiés.

Les résultats présentés dans cette thèse peuvent aussi être utilisés pour déterminer les paramètres optimaux pour un système de rétro-propagation optique fonctionnant dans des conditions imparfaites. 


\section{Acknowledgments}

First and foremost, I would like to express my sincere gratitude to my supervisor Prof. Lawrence R. Chen for his continuous support, encouragement and guidance through my Master study and research, for his motivation, patience and valuable knowledge. Lawrence has helped me not only by providing valuable research assistant but also emotionally and academically through the difficulties to finish this thesis. Without him, this thesis would not have been completed.

Besides my supervisor, I would like to thank the rest of my thesis committees.

My sincere thanks also goes to my colleagues who have helped me with the creation of this work: Jia Li, Meng Qiu, Qunbi Zhuge, Yifan Qi, Mina Spasojevic and all the others that helped on various occasions. Special thanks to Alireza Nezam-Alhosseini who guided me through the beginning of this work and Mohammad Rezagholipour who helped me with the simulation.

I would also like to thank the entire Photonic Systems Group (PSG) for their assistantship and McGill University and NSERC for their support.

Last but not least, I would like to give my sincere thanks to my family members, for their endless support and patience and their accompaniment through the whole process of this thesis.

Guanhui Wang 


\section{Contents}

1 Introduction $\quad 1$

1.1 Motivations for Optical Back Propagation . . . . . . . . . . . . . 1

1.1.1 Wavelength Division Multiplexing \& Advanced Modulation Formats 2

1.1.2 Fiber Nonlinearity and Control . . . . . . . . . . . . . . . . 3

1.1.3 Nonlinearity Control Methods . . . . . . . . . . . . . . . . . . 6

1.2 Optical Phase Conjugation Technique . . . . . . . . . . . . . . . . . . 10

1.3 Motivation and Thesis Objectives . . . . . . . . . . . . . 13

1.3.1 Thesis Outline . . . . . . . . . . . . . . . . . 13

2 OBP Building Blocks $\quad 15$

2.1 OBP Technique \& Working Principles . . . . . . . . . . . . . . . . . 15

2.2 OBP Simulation Set Up . . . . . . . . . . . . . . . . . . . 19

2.2.1 System Setup . . . . . . . . . . . . . . . . . 20

2.2.2 Parameters Setting . . . . . . . . . . . . . . . 20

2.2.3 Ideal OBP Performance and Comparison . . . . . . . . . . . . . . . 22

3 Simulation Results of OBP with Imperfection 26

3.1 OBP Simulation with OSNR Degradation in OPC Process . . . . . . . . . 26

3.2 OBP Simulation with Different in-line Amplifier Noise Figure . . . . . . . 31

4 Simulation Results of OBP that Employs Different Baud Rate and Modulation Format $\quad 35$

4.1 OBP Simulation with Different Baud Rate . . . . . . . . . . . . . . 35

4.2 OBP Simulation with Different Modulation Format . . . . . . . . . . . 37 
5 Conclusion \& Future Work 43

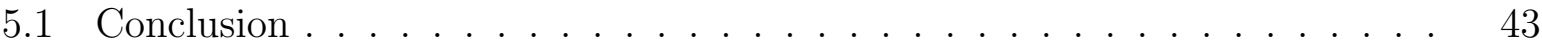

5.2 Future Work . . . . . . . . . . . . . . . . . . . . 44

$\begin{array}{ll}\text { References } & 47\end{array}$ 


\section{List of Figures}

1.1 The evolution of optical fiber transmission capacity . . . . . . . . . . . 1

1.2 Wavelength division multiplexing system scheme diagram . . . . . . . . . . 2

1.3 Constellation diagrams of various modulation formats . . . . . . . . . . . 3

1.4 Shannon-Hartley Theorem \& Information Theorem . . . . . . . . . . . . . 4

1.5 Nonlinear Effect Categories in Optical Fibers . . . . . . . . . . . . . 5

1.6 Maximum Launch Power per Channel v.s. Channel Number . . . . . . . . 5

1.7 DBP performance . . . . . . . . . . . . . . . 7

1.8 OPC transmission reach test for a DWDM DQPSK system . . . . . . . . 8

1.9 Optical fiber communication system with OBP . . . . . . . . . . . . . 10

1.10 SOA based optical phase conjugating system . . . . . . . . . . . . . . . . . 11

1.1190 degree hybrid SOA MZIs OPC scheme . . . . . . . . . . . . . . . . 12

1.12 Wavelength converter based on four-wave-mixing in silicon nanowire experimental results . . . . . . . . . . . . . . . . . . . . . . . . . 12

2.1 Full step-size OBP building Blocks . . . . . . . . . . . . . . . . . . 16

2.2 Half step-size OBP building Blocks . . . . . . . . . . . . . . . . . . . 18

2.3 OBP building Blocks . . . . . . . . . . . . . . . . . . . . 19

2.4 Schematic diagram of the OBP communication system . . . . . . . . . 21

2.5 BER versus launch power. Transmission distance $=800 \mathrm{~km}$. . . . . . . . 23

2.6 Ideal OBP \& OPC performance . . . . . . . . . . . . . . . . . . 24

3.1 OBP with OPC OSNR degradation measurements . . . . . . . . . . . 27

3.2 Power penalty for OBP with imperfect OPC OSNR degradation \& optimized BER and launch power v.s. OSNR degradation . . . . . . . . . . . . 29

3.3 OBP system SNR performance . . . . . . . . . . . . . 30 
3.4 Reach test for OBP with imperfect OPC OSNR degradation . . . . . . . . 31

3.5 Reach percentage under different OSNR degradation for FSS OBP \& HSS

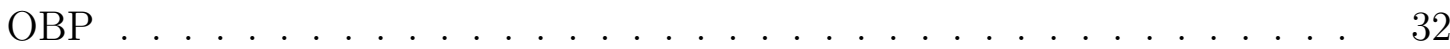

$3.6 \quad$ System performances with in-line amplifier $N F=4.5 d B \& 12 d B \ldots \ldots 33$

4.1 System performances of OBP with $25 \mathrm{Gbaud} / \mathrm{s} \& 28 \mathrm{Gbaud} / \mathrm{s} \ldots \ldots . \ldots 36$

4.2 System performances of OBP with $28 \mathrm{Gbaud} / \mathrm{s}$ for in-line amplifier noise figure of $4.5 d B$ and $12 d B \ldots \ldots \ldots \ldots \ldots$

4.3 System performances of OBP with $32 Q A M$ \& $64 Q A M$ modulated signals . $\quad 39$

4.4 System performances of OBP with in-line amplifier noise figures of $4.5 d B$ \& $12 d B$ for $64 Q A M$ modulated signals . . . . . . . . . . . . . . . 41

5.1 In-line OBP schematic diagram $\ldots \ldots \ldots \ldots$

5.2 In-line nonlinear compensation schematic diagram . . . . . . . . . . 45

5.3 OBP span parameters with variation $\ldots \ldots \ldots . \ldots . \ldots . \ldots 46$ 


\section{List of Tables}

2.1 Parameters Setting for full step-size OBP . . . . . . . . . . . . . . . . 21

2.2 Parameters Setting for half step-size OBP _ . . . . . . . . . . . . 22

2.3 Constellation maps for different configurations under different launch power 25

4.1 Constellation maps for half step-size OBP with 25GBaud under different launch powers for $32 Q A M \& 64 Q A M \ldots \ldots \ldots \ldots \ldots$ 


\section{List of Acronyms}

$\begin{array}{ll}\text { WDM } & \text { Wavelength-Division-Multiplexing } \\ \text { OOK } & \text { On-Off Keying } \\ \text { PSK } & \text { Phase-Shift Keying } \\ \text { QAM } & \text { Quadrature Amplitude Modulation } \\ \text { SNR } & \text { Signal-to-Noise Ratio } \\ \text { SPM } & \text { Self Phase Modulation } \\ \text { XPM } & \text { Cross Phase Modulation } \\ \text { FWM } & \text { Four-Wave-Mixing } \\ \text { SRS } & \text { Stimulated Raman Scattering } \\ \text { SBS } & \text { Stimulated Brillouin Scattering } \\ \text { CIP } & \text { Carrier Induced Phase Modulation } \\ \text { NLSE } & \text { Nonlinear Schrödinger Equation } \\ \text { BP } & \text { Back Propagation } \\ \text { DBP } & \text { Digital Back Propagation } \\ \text { OBP } & \text { Optical Back Propagation } \\ \text { OPC } & \text { Optical Phase Conjugation } \\ \text { OSNR } & \text { Optical Signal-to-Noise Ratio } \\ \text { FEC } & \text { Forward Error Correction } \\ \text { CD } & \text { Chromatic Dispersion } \\ \text { PMD } & \text { Polarization Mode Dispersion } \\ \text { DCF } & \text { Dispersion Compensation Fiber } \\ \text { SOA } & \text { Semiconductor Optical Amplifier } \\ \text { MZI } & \text { Mach-Zehnder Interferometer } \\ \text { DWDM } & \text { Dense Wavelength-Division-Multiplexing } \\ & \end{array}$


HDF Highly Dispersion Fiber

FBG Fiber Bragg Grating

HNLF Highly Dispersion Fiber

TE Transverse Electric

TM Transverse Magnetic

OSSBM Optical Single Sideband Modulator

PC Polarization Controller

BER Bit Error Rate

FSS Full Step-Size

SSF Split-Step Fourier

HSS Half Step-Size

SMF Single Mode Fiber

DSP Digital Signal Processing

SSMF Standard Single Mode Fiber

NF Noise Figure 


\section{Chapter 1}

\section{Introduction}

\subsection{Motivations for Optical Back Propagation}

In the year 1964 when Dr. C. K. Kao first theoretically and critically identified the specifications for long range fiber optics [1], fiber-optic communication became the rising star of high-speed data communication in the information age. Today, more than 80 percent of the long distance traffic worldwide is carried by optical fiber cables [2]. At this information explosion age, the extraordinary large quantity of data requires higher and higher transmission capacity of the communication systems. The transmission capacity shows a growth trend about 10 times in every 4 years as the evolution shown in Fig 1.1 [3].

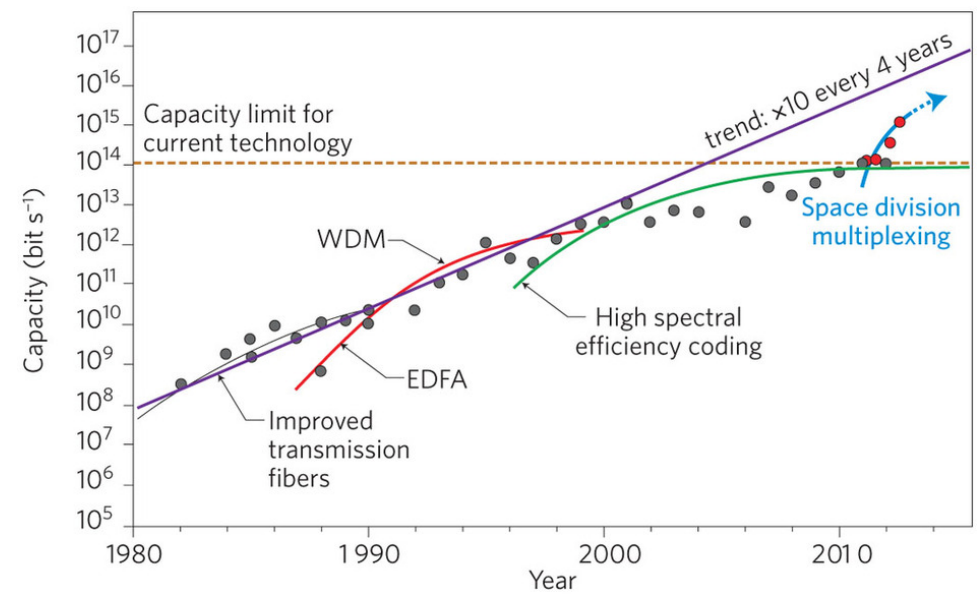

Fig. 1.1 The evolution of optical fiber transmission capacity [3]. 


\subsubsection{Wavelength Division Multiplexing \& Advanced Modulation Formats}

Wavelength division multiplexing (WDM) (Figure 1.2) is one of the promising techniques for increasing transmission capacity in fiber optic communication systems. WDM refers to the technique in which multiple optical carriers at different wavelengths are modulated independently by different electrical bit streams [4]. This technique allows multiple optical signals transmitted simultaneously through the same fiber hence increases the systems transmission capacity significantly. An optical multiplexer can be used to combine the signals and an optical wavelength division demultiplexer can be used to separate the signals. The separated optical signals at different wavelengths can then be sent to different detectors. The WDM technique also allows the exploitation of large bandwidth provided by optical fibers.

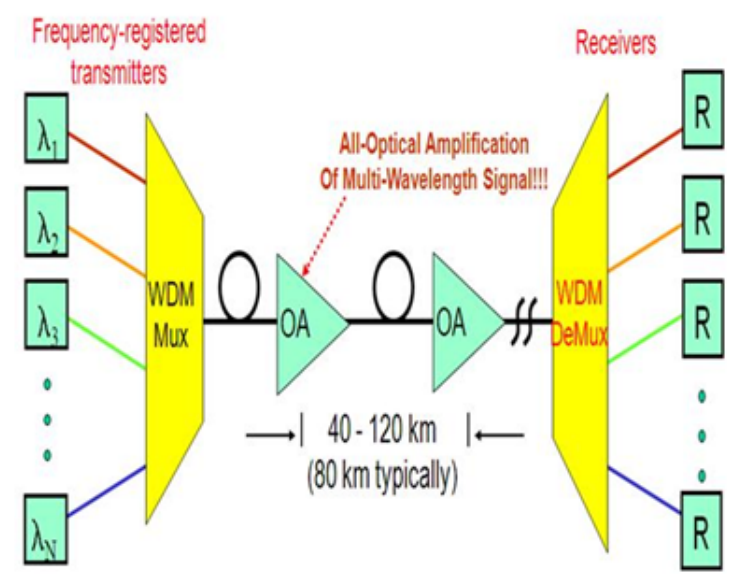

Fig. 1.2 Wavelength division multiplexing system scheme diagram (figure taken from http://1000projects.org/adaptive-reliable-multipath-provisioningin-survivable-wdm-mesh-networks.html)

Another technique that increases the transmission capacity significantly in fiber optic communication systems is advanced modulation formats of optical signals. While the modulations on the lightwave could be made at the space, polarization, frequency and time of the optical signals [5], in this section, the attention would be put on the quadrature modulation formats in which the electrical signal could be modulated on the amplitude or phase information of the lightwave. Besides the basic on-off keying (OOK) modulation, the more spectrally efficient phase-shift keying (PSK) and quadrature amplitude modulated (QAM) modulation formats enable higher transmission bit rate while maintaining 
the same baud rate in the system. Figure 1.3 [6] shows several modulation formats constellation maps including 16QAM and 64QAM, which have 4 bits per symbol and 6 bits per symbol respectively.

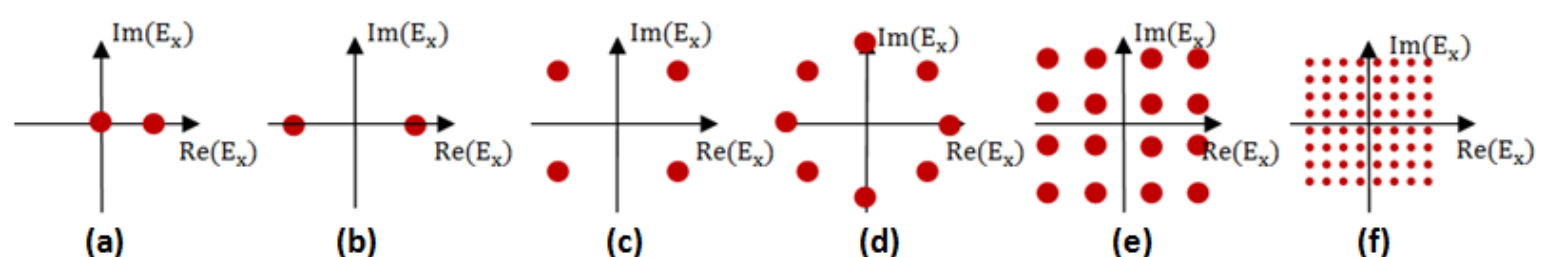

Fig. 1.3 Constellation diagrams of various modulation formats: (a) OOK (b) BPSK (c) QPSK (d) 8PSK (e) 16QAM and (f) 64QAM

\subsubsection{Fiber Nonlinearity and Control}

Figure 1.1 verifies that by using WDM technique and advanced modulation formats, the transmission capacity of fiber optic systems could indeed be increased while there still exists factors that limit the capacity from growing glitch free. The transmission channel capacity could be captured by the Shannon-Hartley Theorem as [7]:

$$
C=B \log _{2}\left(1+\frac{S}{N}\right)
$$

where $B$ is the channel bandwidth and $\frac{S}{N}$ is the signal to noise ratio (SNR). While the Shannon-Hartley Theorem simply suggests that the system channel capacity would increase logarithmically as a function of the system's signal to noise ratio, the growth of the transmission capacity is not realized by increasing the signal launch power into the system. Several problems will arise when the signal power in the optical fiber is high. Figure 1.4 [8] shows the real case when increasing the launch power into the optical fiber, the capacity curve will actually bend down when the signal power is high. There are several reasons why the transmission capacity could not be continuously enlarged by simply increasing channel launch power while the major one is the nonlinearity effect in the optical fiber.

While the Shannon-Hartley Theorem suggests that the channel capacity would increase along with the increasing channel launch power, the nonlinear effects in the optical fiber suggest a bending down of the curve. Nonlinear effect is one of the unique characteristics of 


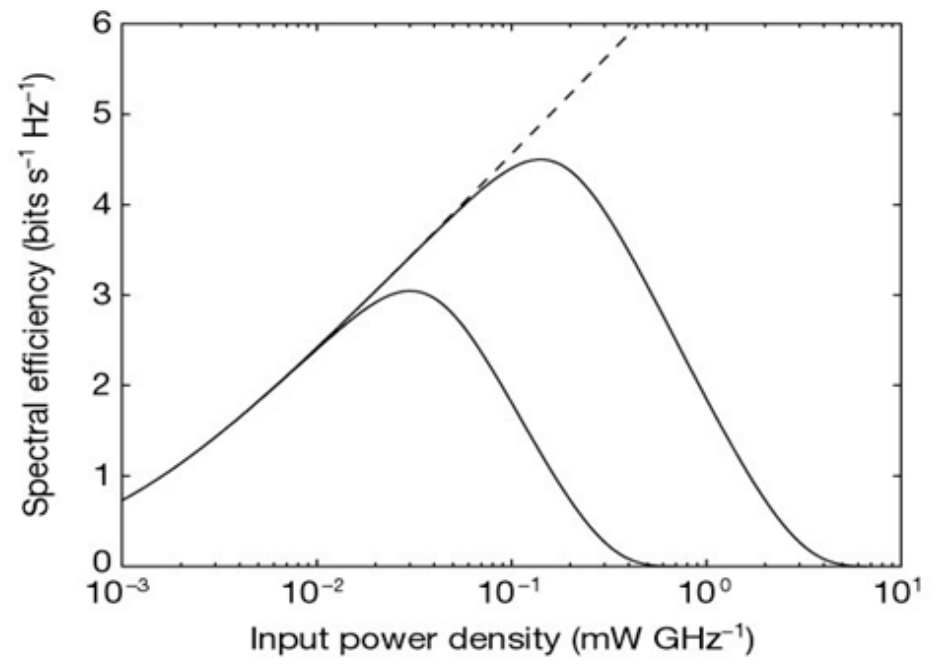

Fig. 1.4 Shannon-Hartley Theorem \& Information Theorem [8]

fiber optic communications. It has been intensively studied on optical fiber based systems. The nonlinear effect is an intensity dependent phenomenon in optical fibers. It occurs due to the refractive index change of the optical fiber with the change of the light intensity, and inelastic scattering in optical media. Though nonlinear effects can cancel out some linear dispersion in optical fibers [9], generally speaking, the nonlinearity in optical fiber limits the performance of fiber communication systems. Nonlinear effects will cause serious drawbacks in fiber optic communication systems, especially in WDM systems. It has been shown that the nonlinear effects cannot be ignored in high-bit-rate WDM systems ( $>10$ Gbit/s per channel). For instance, in a typical commercial 128-channel WDM system, the launch power per channel needs to be maintained under $-5 \mathrm{dBm}$ and the bit rate needs to be maintained under $10 \mathrm{Gbit} / \mathrm{s}$ accordingly in order not to trigger the significant information capacity degradation caused by nonlinearity [10].

There are five nonlinear effects in optical fibers. As shown in Figure 1.5 [11], Self-PhaseModulation (SPM), Cross-Phase-Modulation (XPM) and Four-Wave-Mixing (FWM) are caused by the nonlinear refractive index effects; Stimulated-Raman-Scattering (SRS) and Stimulated-Brillouin-Scattering (SBS) are caused by inelastic scattering in optical fiber media [12]. Each of these nonlinear effects in optical fiber will affect light waves in different ways. Figure 1.6 shows the maximum launch power per channel versus the numbers of channels which ensures SRS, carrier induced phase modulation (CIP), SBS, FWM degra- 


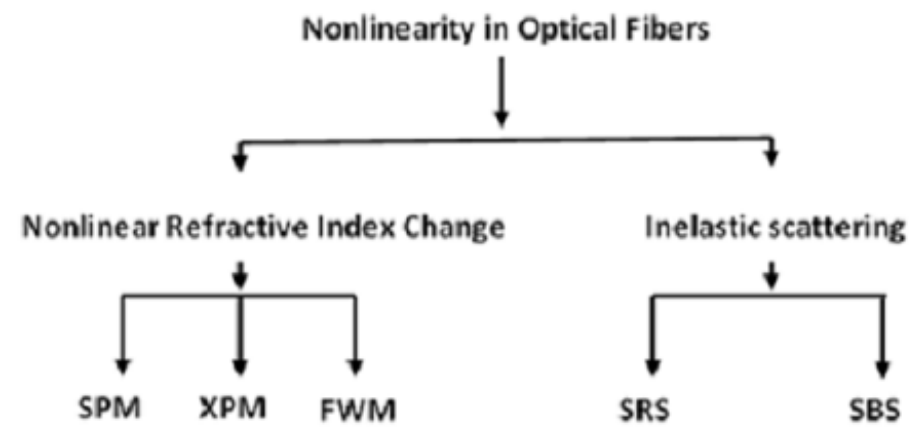

Fig. 1.5 Nonlinear Effect Categories in Optical Fibers

dation below $1 \mathrm{~dB}$ for all channels [10]. The results are for $100 \mathrm{GHz}$ channel spacing WDM system assuming lossless passive multiplexing for $10 \mathrm{~mW}$ and $50 \mathrm{~mW}$ laser transmitters and all the parameters are defined in the figure.

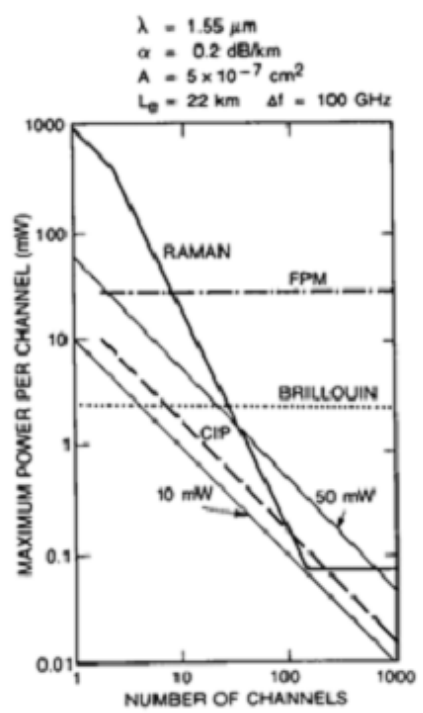

Fig. 1.6 Maximum Launch Power per Channel v.s. Channel Number [10]

If the third order dispersion, XPM and FWM are ignored, the optical field envelope evolution in a fiber optic communication system could be described by the nonlinear Schro dinger equation (NLSE) as [13]: 


$$
\frac{\partial \mu}{\partial z}=j[D(t)+N(t, z)] \mu(t, z)
$$

where

$$
\begin{gathered}
D(t)=-\frac{\beta_{2}}{2} \frac{\partial^{2}}{\partial t^{2}} \\
N(t, z)=\gamma a^{2}(z)|\mu(t, z)|^{2}
\end{gathered}
$$

$\beta_{2}$ is the dispersion coefficient and is the nonlinearity coefficient. The channel loss can be written as:

$$
a^{2}(z)=\exp \left[-\bmod \left(z, L_{a}\right) \alpha\right]
$$

where $L_{a}$ is the amplifier spacing and $\alpha$ is the fiber loss coefficient.

\subsubsection{Nonlinearity Control Methods}

WDM system is one of the promising solutions to high-capacity transmission in optical communication systems. With denser channel spacing, the total transmission rate would be higher while the interferences from other channels caused by nonlinearity would be more severe too. Recently, back-propagation (BP) has been proposed as a promising universal technique to compensate nonlinear and linear impairments in optical communication systems. While digital back propagation (DBP) becomes more mature, optical back propagation $(\mathrm{OBP})$ is currently introduced as an easier and more efficient technique to deal with nonlinearity in optical fibers. Other methods such as mid-link optical phase conjugation (Mid-Link OPC) are also common as nonlinearity control methods. In this section, the three methods and their working principles will be briefly introduced.

\section{Digital Back Propagation}

Provided that the channel characteristics are known, DBP is commonly referred as using split-step inverse NLSE solution to pre-compensate or post-compensate the deterministic effects in channels at the transmitter or receiver [14]. The working principle of DBP is straightforward. By estimating the transmission link as a channel, the channel response can be characterized using a loop back or other methods. Then at the systems receiver 
or transmitter, the inverse channel response can be applied to the signal to recover the dispersions happened in the channel. DBP can jointly compensate linear and nonlinear effects at the same time. By using the digital signal processing of DBP, the launch power in WDM systems can be significantly increased to the values beyond the traditional nonlinear limits. As a result, higher optical signal-to-noise ratio (OSNR) or longer transmission distance which reaches the forward error correction (FEC) limit can be achieved. Figure 1.7 (a) shows the phase error versus launch power with/without DBP. The simulation at the receiver is for a $21.4 \mathrm{~Gb} / \mathrm{s} 50 \%$ RZ-QPSK transmission system over 25 spans of $80 \mathrm{~km}$ single mode fiber with $10 \%$ chromatic dispersion (CD) under-compensation per span. The oversampling rate is 2. Figure 1.7(b) shows the transmission reach comparison results of the same system with the same oversampling rate [15].

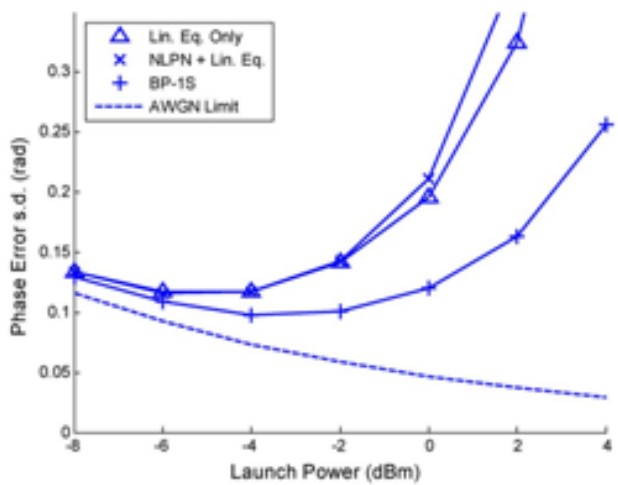

(a)

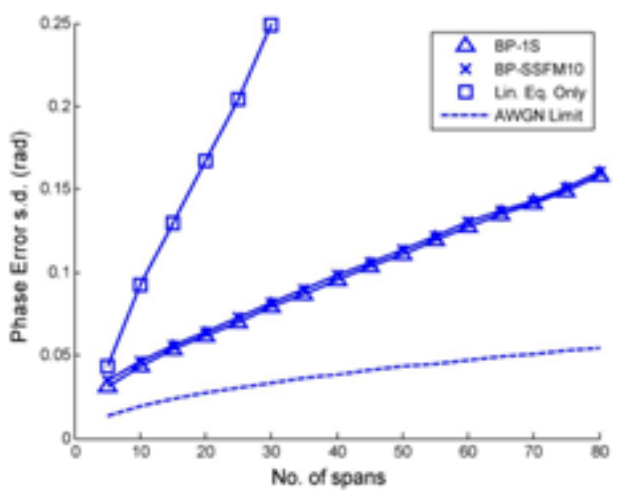

(b)

Fig. 1.7 DBP performance for a 21.4Gb/s 50\% RZ-QPSK transmission system with 10\% CD under-compensation per span. (a) Phase error v.s. launch power, (b) transmission reach test. [15]

Since the NLSE can highly accurately characterize the fiber optic communication systems, the method digital back propagation can accurately compensate the dispersion and nonlinearity in optical fibers. However it still has two non-ignorable drawbacks. DBP is based on the digital signal processing before or after the transmission, which is generally an offline signal processing method that requires excessive computational complexity espe- 
Introduction

cially in WDM systems when considering inter-channel interference. It is also difficult to apply in the presence of polarization mode dispersion (PMD) [16], which means that when using phase modulation and coherent detection, the application of DBP would be difficult.

\section{Mid-Link Optical Phase Conjugation}

Mid-Link OPC is an attractive method to compensate chromatic dispersion and first-order nonlinearity in fiber optical communications without using the chromatic dispersion compensation fiber (DCF). In Mid-Link OPC, an optical phase conjugator is placed in the middle of the transmission link, and then the transmission signal spectrum is inverted. The first-order phase impairments accumulated in the first half of the link can then be counteracted in the second half. The technique of phase conjugation can be achieved by using four-wave-mixing in semiconductor optical amplifiers (SOA) [17] or by using a 90 degree optical hybrid and nested SOA Mach-Zehnder interferometers (SOA MZIs) [18]. Details will be given in later chapters. The demonstrated Mid-Link OPC performance in a dense wavelength division multiplexing (DWDM) $2 \times 10.7 G$ Bits / s DQPSK system is given in Figure 1.8, compared to using DCF alone [19].

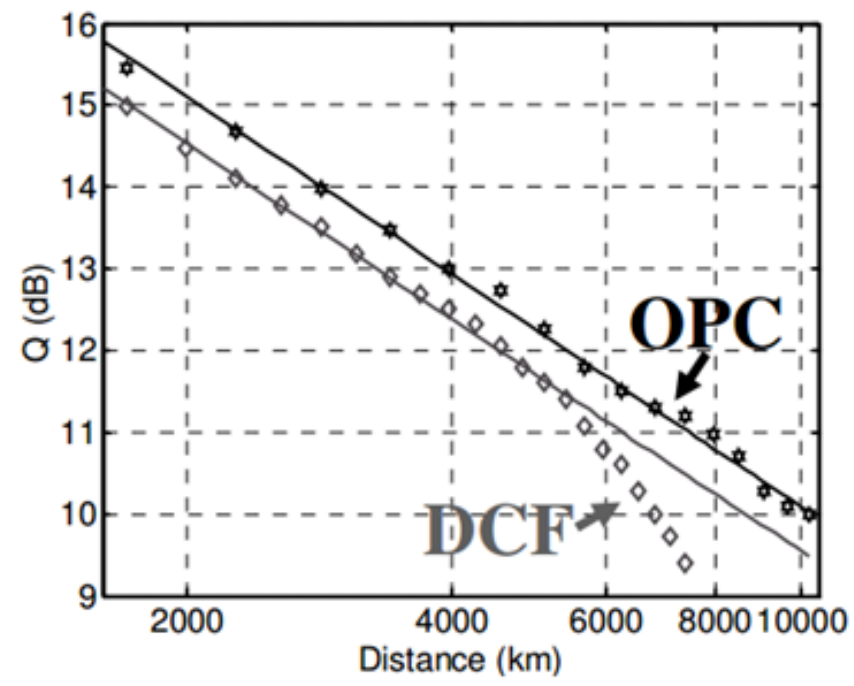

Fig. 1.8 OPC transmission reach test for a DWDM $2 \times 10.7 G B i t s / s$ DQPSK system [19]

The principle of Mid-Link OPC is straightforward, but the implementation of it faces two difficulties. One major concern for this method being used in the industry is that, when 
there are different routes in the transmission systems, the mid-point for different routes are different, and then it is hard to be determined. Another concern is that, in a long haul fiber optic communication system, ensuring that the first and second halves of the transmission link are identical cannot be guaranteed, and then the unsymmetrical power profile with respect to the location of the phase conjugator would degrade the compensation effect. Given these drawbacks of Mid-Link OPC, it is rarely used in the real long haul systems for nonlinearity compensation.

\section{Optical Back Propagation}

Since the midpoint is difficult to be defined in a long haul communication system with different routes, the receiver based optical back propagation is developed to overcome this problem and at the same time it can take the advantage of OPC. In the optical back propagation system, the received signal output from the transmission link would first go through an optical phase conjugator which is followed by a block of optical back propagation schemes before it gets into the photo detector. The OPC inverts the optical spectrum of the transmission link output signal and the OBP can then counteract the phase shifts of the signal which accumulated in the transmission link. The OBP scheme employs highly dispersion fibers (HDFs) or fiber Bragg gratings (FBGs) for chromatic dispersion compensation and highly nonlinear fibers (HNLFs) for nonlinearity compensation [20]. Figure 1.9 [13] shows the schematic diagram of a fiber optic communication link with OPC and OBP. One thing worth to be mentioned is that, the receiver of the system could be a direct detection receiver or a coherent receiver, which means that phase modulation could be used in the system and polarization mode dispersion does not have serious effects on OBP performance since it could be compensated by using digital signal processing afterwards. For all the simulations in this thesis, the signals are quadrature amplitude modulation (QAM) modulated and a coherent receiver is used.

The deduction in [13] shows that, the initial transmission field envelope would be recovered at the OBP output if the transfer function of OBP is the inverse of that of the transmission link. Let $L_{\text {total }}$ be the total length of the transmission link, if the solution of (1.2) written as the form [21]:

$$
\mu\left(t, L_{\text {total }}\right)=M \mu(t, 0)
$$




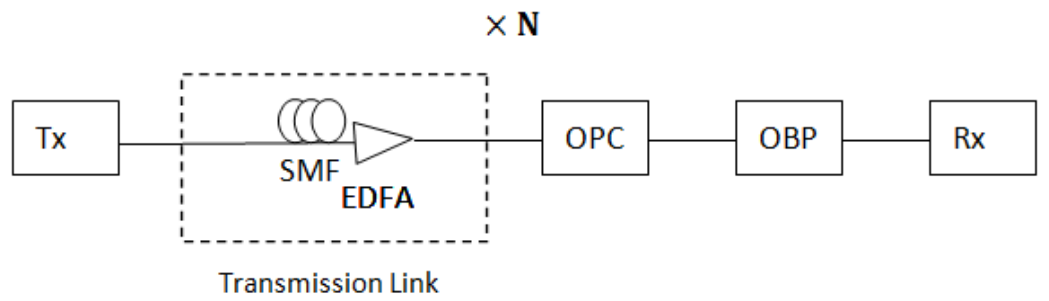

Fig. 1.9 Optical fiber communication system with OBP

$$
M=e^{j \int_{0}^{L_{t o t a l}}[D(t)+N(t, s)] d s}
$$

We can easily get that:

$$
M^{-1}=e^{-j \int_{0}^{L_{t o t a l}}\left[D(t)+N\left(t, L_{t o t a l}-s\right)\right] d s}
$$

Then the output of the OBP would be

$$
\mu_{O B P, \text { out }}=M^{-1} \mu^{*}\left(t, L_{\text {total }}\right)=\mu(t, 0)
$$

which is exactly the original field we want to recover and $\mu^{*}\left(t, L_{\text {total }}\right)$ is the complex conjugate of the fiber transmission link output.

\subsection{Optical Phase Conjugation Technique}

One of the key elements in the optical back propagation scheme is the optical phase conjugator. References [22-27] have discussed the detailed working principles and some experimental set ups to actualize OPC. There are several ways to do an all-optical phase conjugation in an in-line fiber-optic system. The most common OPC techniques are by using four-wave-mixing in semiconductor optical amplifier or 90 degree hybrid and nested semiconductor optical amplifier Mach-Zehnder interferometers.

Figure 1.10 shows the experimental setup for optical phase conjugator using four-wavemixing in semiconductor optical amplifiers, the counter-propagating conjugate wave would be generated. The measured maximum and minimum SOA spontaneous emission spectrum after the adjustable linear polariser were identified as the transverse electric (TE) mode and 


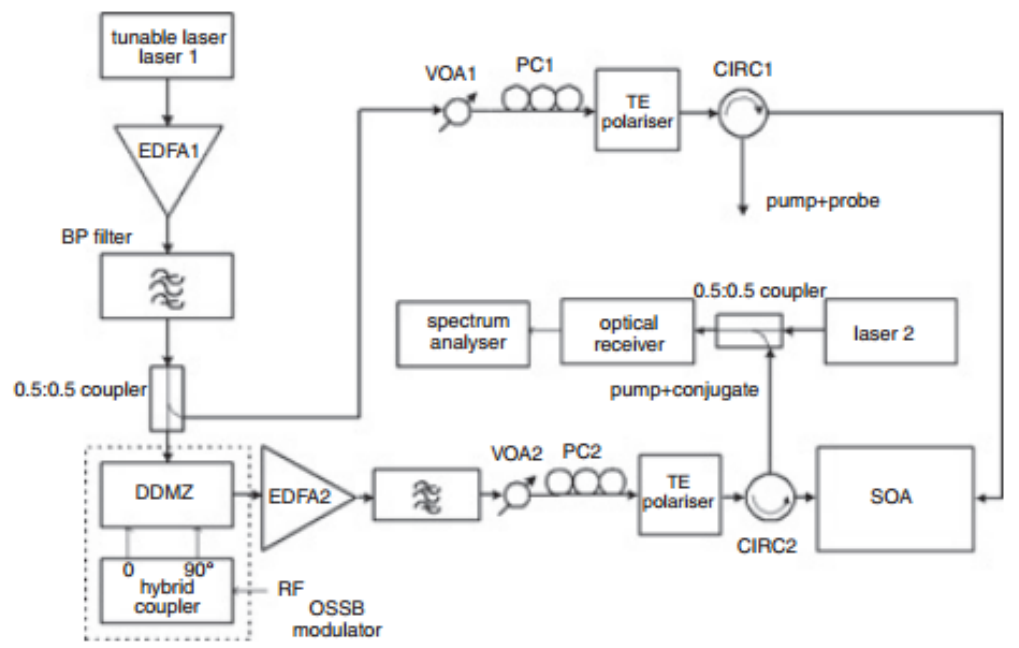

Fig. 1.10 SOA based optical phase conjugating system [17]

the transverse magnetic (TM) mode of the SOA. Laser 1 is a tunable external pump laser and laser 2 is a highly coherent laser. The laser 1 output is amplified by an EDFA to boost the power and then filtered by a band-pass filter to cut off noise and split by a $3 d B$ coupler. One of the $3 d B$ coupler outputs is fed into an optical single sideband modulator (OSSBM) to generate very small frequency detuned (nearly degenerate regime) pump-probe waves with the same state of polarization. Then the modulated wave is boosted and filtered again, the signal power is final controlled by the attenuator (VOA1). Finally, by using the adjustable linear polariser, the co-propagating plump and the counter-propagating pump and the probe waves are aligned to the orthogonal TE and TM modes of the SOA. Polarization controller (PC) 1 and 2 are used to couple the light waves states of polarization. The pump wave is injected through circulator 1 (CIRC1) and the probe plus pump waves are injected through CIRC2.

The conjugate signal output is difficult to measure since the pump-probe frequency difference is small. In order to have an effective optical frequency resolution of the output, the conjugate wave and the pump are mixed with the highly coherent laser 2 which has a frequency offset of $4 G H z$ from the pump laser. To avoid the photodiode nonlinearity, the input power to the receiver should be kept low [17].

Figure 1.11 shows the 90 degree hybrid SOA MZIs OPC scheme based on cross-phase 


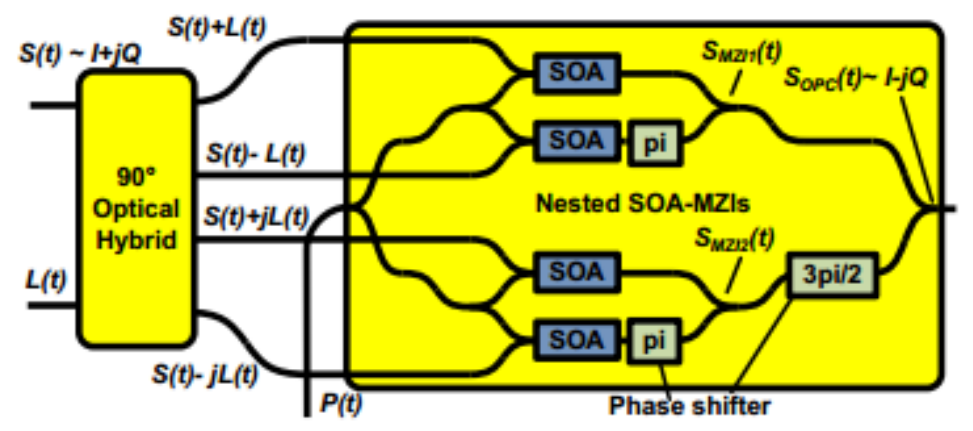

Fig. 1.1190 degree hybrid SOA MZIs OPC scheme [18]

modulation and coherent wave mixing, no parametric process in nonlinear media in this configuration is needed. The phase conjugated wave can be easily separated by a band-pass filter at the output. The detailed working principles deduction and experimental results are shown in [18].

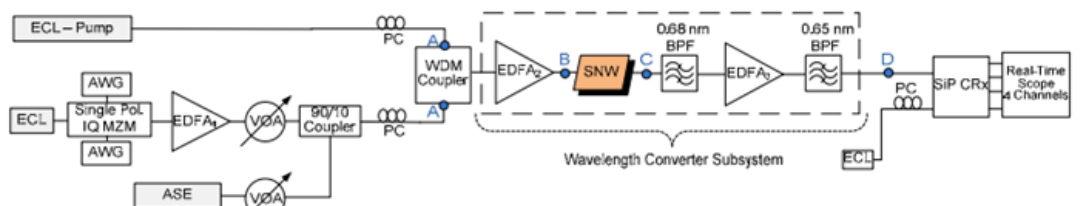

(a)

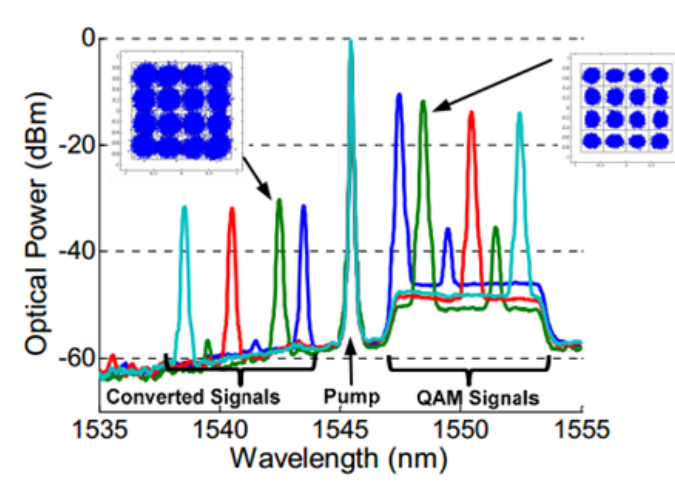

(b)

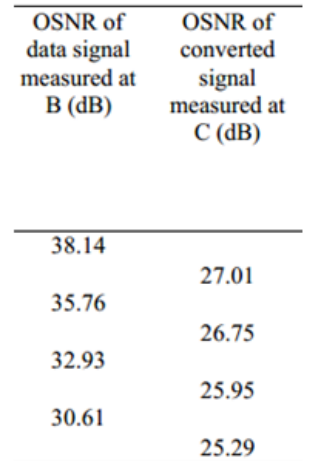

(c)

Fig. 1.12 (a) Wavelength converter based on four-wave-mixing in silicon nanowire experimental setup, (b) Optical spectra of pump, $16-Q A M$ and converted signals and (c) OSNR performances of wavelength conversion right before and after the converter [28]

Although there are a number of schemes that can actualize OPC, in all those techniques, 
the OSNR of the signal at the output will be degraded. The experimental results in [28] and [29] clearly show that, the OPC comes with several up to dozens decibels (dBs) of OSNR degradation. Figure 1.12 shows a wavelength conversion based on four-wave-mixing in silicon nanowire experimental setup and its results for 28GBaud 16QAM signals [28]. The wavelength conversion shares the same technique with OPC and the converted signal experienced an OSNR degradation about $5 d B$ to $10 d B$ depending on the input signals OSNR.

\subsection{Motivation and Thesis Objectives}

In all OBP studies performed to date, ideal conditions are assumed and there have been no studies that investigate the impact of impairments such as OSNR degradation due to the OPC process or due to amplifier noise in the compensation spans. Given that the OSNR degradation is being observed in all experimental OPC process and the in-line amplifier would add noise to the system, it is useful and necessary to quantify these effects on OBP performance.

This thesis will demonstrate the simulation results of optical back propagation with the consideration of different imperfections. The $25 \mathrm{GBaud} / \mathrm{s} 32 Q \mathrm{AM}$ ideal system is used as the based system and all the simulation results are compared to this one. The performance of two different schemes (full step-size and half step-size) of OBP will also be studied. This thesis will also demonstrate the simulation results of OBP with/without imperfection of different baud rates and modulation formats. Higher baud rate (28Gbaud) and higher modulation format $(64-Q A M)$ are employed. The simulations show that if the OSNR of the OPC signal is degraded by $5 d B$, a power penalty as large as $5.7 d B$ will be incurred. Moreover, the transmission distance to obtain a bit error rate (BER) below the forward error correction (FEC) limit will be reduced by around 60 percent. These results can also be used to determine the optimum operating system parameters under non-ideal conditions.

\subsubsection{Thesis Outline}

This thesis is divided into 5 chapters and the remainder organised as follows.

\section{Chapter 2: OBP Building Blocks}

This chapter will introduce the background information of optical back propagation, its 
detailed working principles together with the simulation set up in a fiber optic communication system. Simulation results of ideal systems will be given and compared to simulation results reported previously. All the simulation parameters and condition assumptions will also be given and discussed.

\section{Chapter 3: Simulation Results of OBP with Imperfection}

This chapter will discuss the simulation results of OBP systems when considering the imperfection in the OPC process. The simulated effects of the OSNR degradation after the OPC process on the systems performances will be given and analysed. The effects of the in-line amplifier noise figure on the OBP systems performances will also be studied.

Chapter 4: Simulation Results of OBP that Employs Different Baud Rate and Modulation Format.

This chapter will demonstrate the simulation results of OBP systems with the signals that employ different baud rates and modulation formats. The simulation results of $28 G$ Baud and $64 Q A M$ OBP systems will be provided and discussed. Moreover, the effects of imperfections on these systems will also be given and discussed. A summary of all the simulations will be given.

\section{Chapter 5: Future Work and Conclusion}

This chapter will summarize the work done in this thesis followed by a discussion of the future steps that can be taken toward this work.

The results of this thesis were presented in part in the following conference paper: $\mathrm{G}$. Wang, M. Rezagholipour Dizaji, and L. R. Chen, "Analysis of optical back propagation under non-ideal optical phase conjugation and amplifier noise," Photonics North, 28-30 May 2014, Montreal, QC. 


\section{Chapter 2}

\section{OBP Building Blocks}

\subsection{OBP Technique \& Working Principles}

The receiver based optical back propagation employs similar working principles as mid-link optical phase conjugation whereas it does not counteract the nonlinearity and dispersion phase shifts in the transmission link itself but at the receiver. In optical back propagation, the signal goes through a normal transmission link and right before it gets into the photodetector, the signal undergoes into an OPC block and an OBP block for nonlinearity compensation. The nonlinear and dispersion phase shifts accumulated in the transmission link will be compensated in the back propagation process by inverse the scattering, same as in Mid-Link OPC. Figure 2.1 [13] shows the scheme of a full step-size (FSS) OBP block, it contains a pre-amplifier followed by $\mathrm{N}$ spans of cascaded highly dispersion fibers (HDFs) and highly nonlinear fibers (HNLFs) and in-line amplifiers, where $\mathrm{N}$ is the same number of spans as in the transmission link. The first span in OBP compensates the phase shift of the Nth span in the transmission link. All the numerical characteristics for the HDFs and HNLFs need to be carefully defined so that the nonlinearity effects can be exactly counteracted. One method for calculating these parameters is to partially solve the NLSE.

The NLSE is a nonlinear partial differential equation which does not usually lend itself to numerical analytic solutions. However when the inverse scattering method is employed, a number of numerical approaches of solving NLSE in specific cases are developed. One extensively used method to solve the pulse propagation in nonlinear fibers is the split-step Fourier (SSF) method [30]. This method is also adopted when defining the characteristics for HDFs and HNLFs used in OBP. 


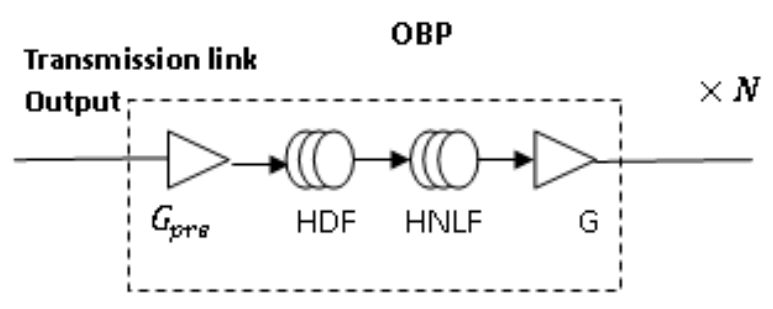

Fig. 2.1 Full step-size OBP building Blocks

To set the parameters for HDFs and HNLFs, we define the electric field in the transmission link fiber to be $\mu(t, z)$ as before, and the electric field in the back propagation to be $\mu_{b}(t, z)$. Similar as equation (1.2), we have the back propagation pulse envelope propagating in the fiber as [31]:

$$
\frac{\partial \mu_{b}}{\partial z}=j\left[D(t)+N\left(t, L_{t o t a l}-z\right)\right] \mu_{b}(t, z)
$$

By the phase conjugation, we have

$$
\mu_{b}(t, 0)=\mu^{*}\left(t, L_{\text {total }}\right)
$$

Take the complex conjugate of equation (1.9) we have

$$
\begin{aligned}
\mu_{O B P, \text { out }}^{*}(t) & =e^{j \int_{0}^{L_{\text {total }}}\left[D(t)+N\left(t, L_{\text {total }}-s\right)\right] d s} \mu\left(t, L_{\text {total }}\right) \\
& =M^{\prime} \mu\left(t, L_{\text {total }}\right) \\
& =\mu^{*}(t, 0)
\end{aligned}
$$

Apply the split-step Fourier technique with a step size of $\Delta z$

$$
M^{\prime} \approx A(t) \bullet B(t, \Delta z) \bullet A(t) \bullet B(t, 2 \Delta z) \bullet \ldots A(t) \bullet B\left(t, L_{\text {total }}\right)
$$

where

$$
A(t)=\exp [j D(t) \bullet \Delta z]
$$




$$
B(t, x)=\exp \left[j \int_{x-\Delta z}^{x} \gamma a^{2}\left(L_{a}-z\right)\left|\mu_{b}(t, z)\right|^{2} d z\right]
$$

Typically, $\left.\mu_{b}(t, z)\right|^{2}$ varies much slower than $a^{2}(z)$ and hence can be approximate as independent of the transmission distance position $z$. Then equation (2.3) can be evaluated as

$$
\begin{gathered}
B(t, x)=\exp \left\{-j \gamma \Delta z_{e f f}(\Delta z) \times \exp \left[-\alpha L_{a}+\bmod \left(x, L_{a+}\right) \alpha\right]\left|\mu_{b}(t, z)\right|^{2}\right\} \\
z_{e f f}(\Delta z)=\frac{m\left[1-e^{-\alpha L_{a}}\right]}{\alpha}+\frac{1-e^{-\bmod \left(\Delta z, L_{a}\right) \alpha}}{\alpha}
\end{gathered}
$$

where $m=$ floor $\left(\Delta z / L_{a}\right)$. In equation (2.3), the HDFs can estimate the operator $A$ and the HLNFs estimate the operator $B$ [32]. If the HDFs have zero nonlinearity, the HNLFs would introduce a nonlinear phase shift which is the same as that of the transmission link with the fiber length of $\Delta z$. In this case, we have:

$$
\begin{gathered}
\mu_{H N, \text { out }}=\mu_{H N, i n} e^{j \gamma_{H N} L_{H N, \text { eff }\left|\mu_{H N, i n}\right|^{2}}} \\
L_{H N, \text { eff }}=\frac{1-\exp \left(-\alpha_{H N} L_{H N}\right)}{\alpha_{H N}}
\end{gathered}
$$

where $\alpha_{H N}, \gamma_{H N}$ and $L_{H N}$ are the loss coefficient, nonlinear coefficient and the length of the HNLF respectively.

Lets first consider the case $\Delta z=L_{a}$, where the back propagation step-size equals the amplifier spacing (the length of one span in the transmission link), this case can be defined as full step-size OBP. We define the nonlinear phase shifts of each piece of the HDF, HNLF and the transmission fiber to be $\varphi_{H D F}, \varphi_{H N L F}$ and $\varphi_{T x}$ respectively, the corresponding length of each piece of fiber in one span to be $L_{H D F}, L_{H N L F}$ and $L_{T x}$ respectively. If the nonlinear phase shift in the $j t h$ span in transmission link is fully counteracted by the $(N-j+1)$ th span back propagation, the phase shifts should satisfy the relation:

$$
\varphi_{H D F}+\varphi_{H N L F}=\varphi_{T x}
$$

All the nonlinear phase shifts can be calculated as: 


$$
\varphi_{i}=\gamma_{i} P_{i} L_{i, e f f}
$$

where

$$
L_{i, e f f}=\frac{1-\exp \left(-\alpha_{i} L_{i}\right)}{\alpha_{i}}
$$

is the effective length of fiber type $i$ and $i=H D F, H N L F, T x . P_{i}$ is the launch power to the fiber type $i$ and $\alpha_{i}$ is the loss coefficient. All the parameters can be set by solving equations (2.10), (2.11) and (2.12) given that the parameters for the transmission link are known. Next, we consider the case when $\Delta z=L_{a} 2$, the back propagation step-size equals half of the amplifier spacing (half of the length of one span in the transmission link) and this case can be defined as half step-size (HSS) OBP. Figure 2.2 [13] shows the configuration of half step-size OBP.

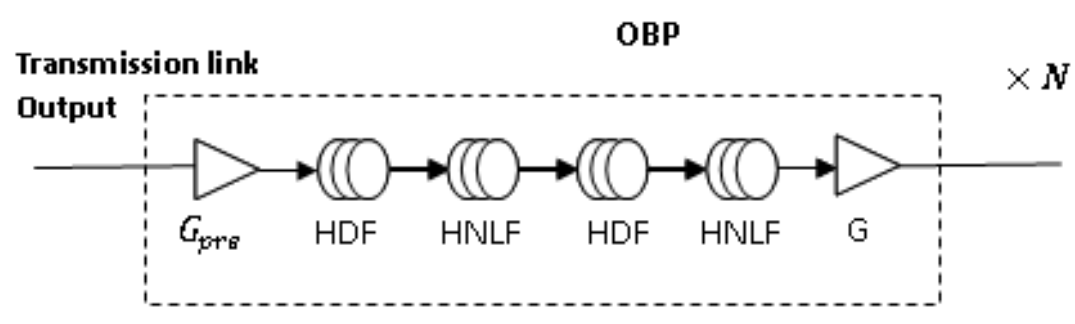

Fig. 2.2 Half step-size OBP building Blocks

The only difference between half step-size OBP and full step-size OBP is that, in half step-size OBP, the first HDF and HNLF set in the back propagation span compensates the second half span of the transmission links nonlinear effect, then the second set of HDF and HNLF compensates the nonlinear effect for the first half span in the transmission link. Here we can choose to use two in-line amplifiers in each OBP span after each set of the HDF and HNLF link or we can use one amplifier at the end of the total span to compensate the total fiber loss as shown in Figure 2.2. The characteristics of HDFs and HNLFs in the half step-size OBP could also be calculated based on the same deduction methods used for full step-size OBP only the step size $\Delta z=L_{a} / 2$. Though the computational complexity to determine the parameters for half step-size OBP is slightly higher than the full step-size one, the compensation results theoretically would be better when the split-step is smaller. 


\subsection{OBP Simulation Set Up}

Given that the information of the transmission links are known, the characteristics of the HDFs and HLNFs in the OBP spans can be theoretically defined. To simplify the work and without loss generality, we consider the nonlinearity contribution from the HDFs to be zero, all the nonlinear effects in the transmission link will be compensated by the HNLFs. Then the number of HDF in each OBP span will not affect the nonlinear effect compensation. The chromatic dispersion accumulated phase shift in the transmission span would be fully counteracted if the dispersion coefficient and fiber length product in the transmission span matches that in the back propagation span.

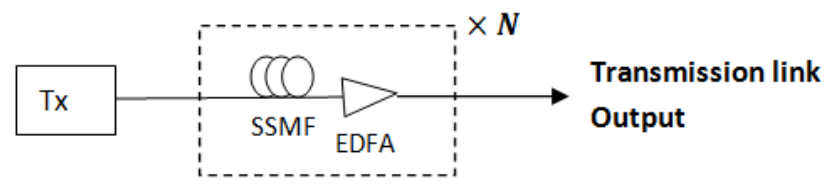

(a)

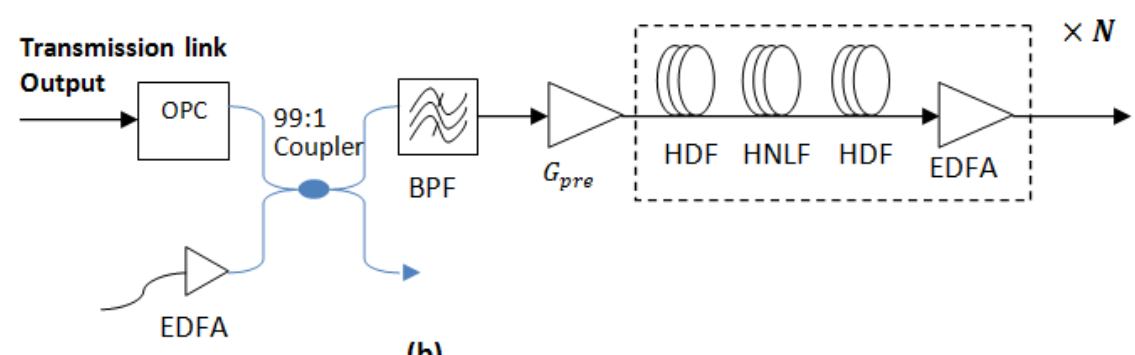

(b)

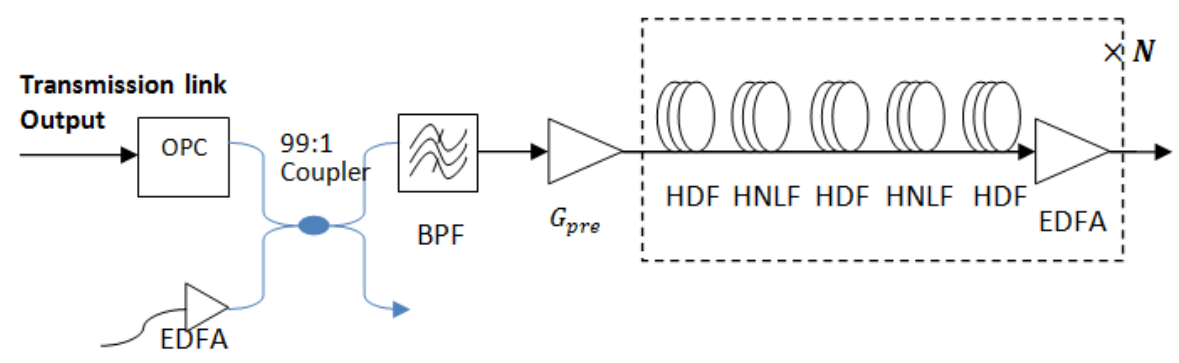

(c)

Fig. 2.3 (a) Optical fiber transmission link simulation set up, (b) full step-size OBP simulation set up and (c) half step-size OBP simulation set up

Figure 2.3 (a) shows the transmission link with $\mathrm{N}$ spans of transmission fibers, (b) 
shows the configuration of the full step-size OBP and (c) shows the configuration of the half step-size OBP. The modulated optical signal first goes through N spans of single mode fibers (SMFs) followed by an in-line amplifier in each span to fully compensate the fiber loss in that span. After the traditional transmission link, the output signal spectrum is inverted by an optical phase conjugator and then coupled with wide range Gaussian white noise by a $99: 1$ optical coupler to boost the noise floor so that it can estimate the OSNR degradation caused by the OPC process. The degraded signal then goes through a bandpass filter to cut off out band spontaneous emission noise and get pre-amplified before it goes into the back propagation. The ideal case simulation can be achieved by simply turn off the EDFA input to the coupler. Then in the back propagation spans, the cascaded HDFs and HNLFs counteract the CD and nonlinear effects caused phase shifts respectively. The system output could then be detected by the photodetector and does the followed up digital signal processing (DSP).

\subsubsection{System Setup}

The simulation of the OBP system is realized using a combination of OptiSystem and MATLAB. The modulated optical signals are generated in MATLAB and imported to OptiSystem. The whole transmission system from the transmitter to the receiver is built up in OptiSystem. The signals are QAM and dual-polarized modulated and therefore a coherent receiver is employed. The coherent receiver can detect not only the amplitude but also the phase and polarization of the received data, hence it can achieve high efficiency modulation of the signals and high data rate. Then the data collected at the receiver is exported and loaded to MATLAB again to do the digital signal processing. Figure 2.4 shows the schematic diagram of this optical communication system. The OBP process compensates the chromatic dispersion effect and nonlinear effect accumulated in the transmission link, other dispersions such as PMD and frequency offset are compensated using DSP in MATLAB.

\subsubsection{Parameters Setting}

To fully compensate CD and nonlinearity in the transmission link, the parameters of the HDFs and HNLFs in OBP links need to be carefully defined. For the ideal case, assume perfect OPC (no OSNR degradation after the phase conjugation) and ideal in-line amplifier 

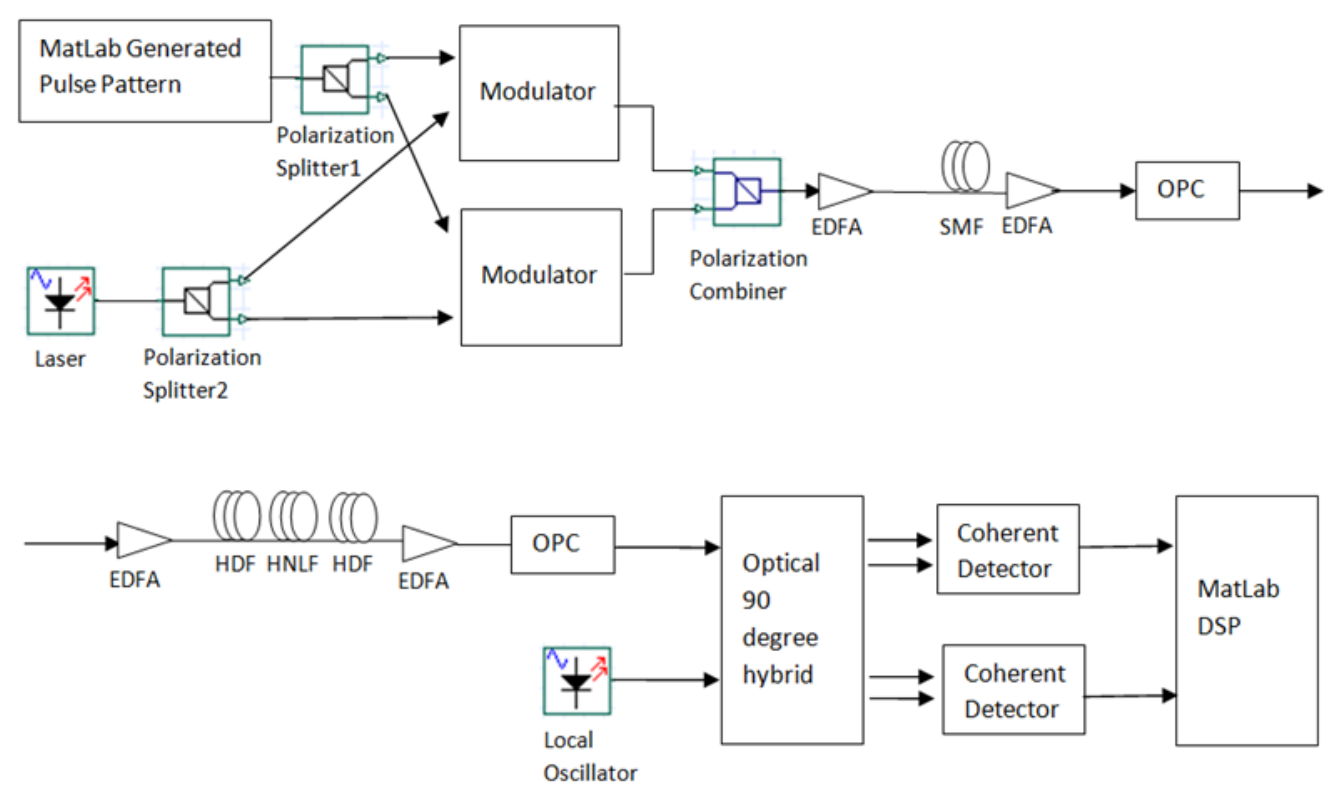

Fig. 2.4 Schematic diagram of the OBP communication system

noise figure $\left(n_{s p}=3\right)$ in the back propagation links. The nonlinear effect of the HDFs are ignored hence the nonlinearity is fully compensated by the HNLFs. Assume that there is no chromatic dispersion in the HNLF. Then the phase shifts caused by CD and nonlinearity in the transmission link are compensated by the HDF and HNLF in back propagation respectively. Table 2.1 shows the parameters setting for the full step-size OBP for the transmission link, HDFs and HNLFs, where $L_{a}$ is the length of the fibers per span and $N$ is the number of spans.

Table 2.1 Parameters Setting for full step-size OBP
Transmission link

HDFs

$80 \mathrm{~km}$
$-21 p \mathrm{~s}^{2} / \mathrm{km}^{-1}$
$2.2 W^{-1} \mathrm{~km}^{-1}$
$0.2 \mathrm{~dB} / \mathrm{km}$
10

HNLFs

$L_{a}$
$\beta_{2}$
$\gamma$
$\alpha$
$\mathrm{N}$

$L_{a}$

$\gamma$

$\alpha$

$\mathrm{N}$

\begin{tabular}{ll|}
$4.5 \mathrm{~km}$ & $70.445 \mathrm{~cm}$ \\
$-186.67 \mathrm{ps}^{2} / \mathrm{km}$ & 0 \\
0 & $2000 \mathrm{~W}^{-1} \mathrm{~km}^{-1}$ \\
$0.4 \mathrm{~dB} / \mathrm{km}$ & $0.3 \mathrm{~dB} / \mathrm{m}$ \\
10 & 10
\end{tabular}

For the full step-size OBP, the phase inversed signal first got boosted by the preamplifier before it is launched into the OBP fibers. The pre-amplifier has a gain of $G_{\text {pre }}=$ 
$14 d B$. With the in-line amplifier fully compensates the fiber loss for each span in the back propagation, it has a gain of $G=3.8 d B$.

\begin{tabular}{c|llll} 
& Transmission link & HDFs & HNLF_1 & HNLF_2 \\
\hline$L_{a}$ & $80 k m$ & $4.5 \mathrm{~km}$ & $20.36 \mathrm{~cm}$ & $78.76 \mathrm{~cm}$ \\
$\beta_{2}$ & $-21 \mathrm{ps}^{2} / \mathrm{km}^{2}$ & $-124.44 \mathrm{ps}^{2} / \mathrm{km}$ & 0 & 0 \\
$\gamma$ & $2.2 W^{-1} \mathrm{~km}^{-1}$ & 0 & $2000 W^{-1} \mathrm{~km}^{-1}$ & $2000 \mathrm{~W}^{-1} \mathrm{~km}^{-1}$ \\
$\alpha$ & $0.2 \mathrm{~dB} / \mathrm{km}$ & $0.4 \mathrm{~dB} / \mathrm{km}$ & $0.3 \mathrm{~dB} / \mathrm{m}$ & $0.3 \mathrm{~dB} / \mathrm{m}$ \\
$\mathrm{N}$ & 10 & 20 & 10 & 10
\end{tabular}

Table 2.2 Parameters Setting for half step-size OBP

Table 2.2 shows the parameters setting for the half step-size OBP, the pre-amplifier gain remains the same as $G_{p r e}=14 d B$. With the in-line amplifier fully compensates the fiber loss for each span in the back propagation, it has a gain of $G=5.7 \mathrm{~dB}$. The lengths of the two segments of HNLFs used here are optimised for the split-step NLSE to have the most accurate solution.

All the parameters are calculated based on the working principles described earlier in this chapter, while the solutions are not unique for both the full step-size and half step-size cases, other sets of parameters could also be used in the simulation.

\subsubsection{Ideal OBP Performance and Comparison}

In [13], the simulation results demonstrated that the optical back propagation at receiver outperforms DBP [33,34] and Mid-link OPC [35-37] as shown in Figure 2.5. 25GBaud $32 Q A M$ signal was adopted in the simulation with a total transmission distance of $800 \mathrm{~km}$. It is shown that at low launch power, the four configurations almost perform equally while when the launch power is higher than $0 \mathrm{dBm}$, the advantage of OBP starts to show up and when the launch power is even higher than $4 \mathrm{dBm}$, half step-size OBP outperforms the full step-size one.

We verified the comparison of OBP and Mid-Link OPC with our simulation systems and the results are shown in Figure 2.6. Figure 2.6(a) is the BER performance as a function of the system launched power over $800 \mathrm{~km}$ of transmission distance and (b) is the transmission reach performance test.

For the three different systems, the OBP configurations (both full and half step-size ones) have better performance compared with Mid-Link OPC, both in the power test and 


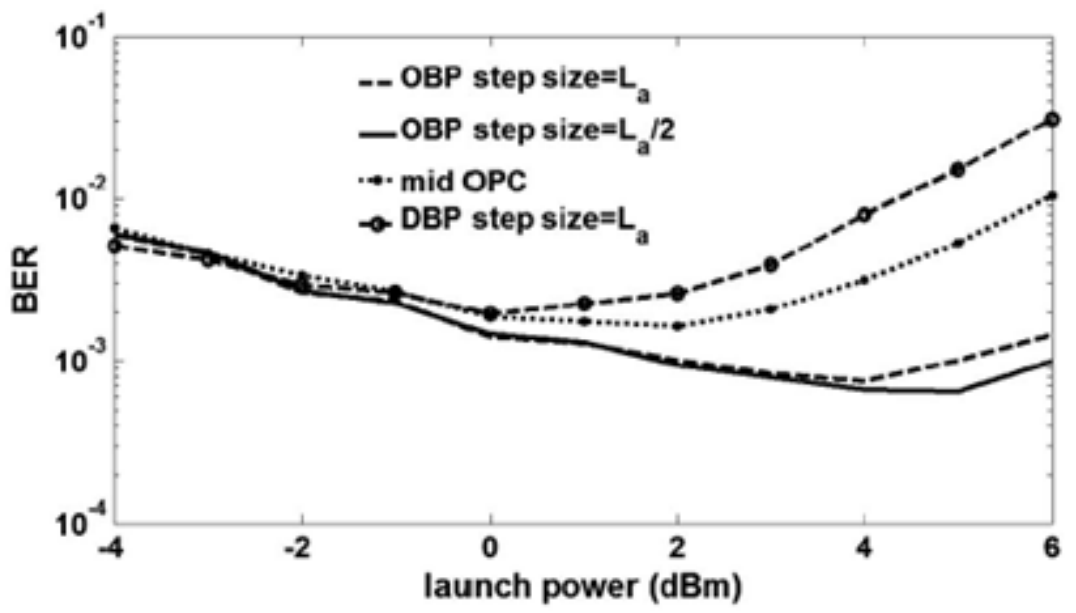

Fig. 2.5 BER versus launch power. Transmission distance $=800 \mathrm{~km}[13]$

the transmission reach test. For different OBP configurations, the full and half step-size OBP almost have the same performance under low system launch power (i.e., lower than $3 \mathrm{dBm}$ ), then along with the increment of the launch power, the bit error rate of full step-size OBP system grows faster. The transmission reach performance test is performed under the situation that the optimized launch power is used in all the configurations (i.e., $4 \mathrm{dBm}$ for full/half step-size OBP and $5 \mathrm{dBm}$ for Mid-Link OPC). The error free recovery transmission distance for Mid-Link OPC is $1560 \mathrm{~km}$ and for full/half step-size OBP are $1720 \mathrm{~km}$ and $2272 \mathrm{~km}$ respectively.

Table 2.3 shows the constellation maps of the three configurations under different launch powers. There is a clear sign of the systems performances difference between Mid-Link OPC and $\mathrm{OBP}$, and at $5 \mathrm{dBm}$ of launch power, the difference between full and half step-size OBP can be observed.

To summarize, this chapter discussed the working principle of OBP, showed its simulation platform in OptiSystem and demonstrated the ideal case simulation results. The results showed that under ideal cases, OBP outperforms DBP and Mid-Link OPC. MidLink OPC has a tendency of outperforming OBP when the transmission distance is further increased in the ideal case simulation, which makes sense since in the ideal case simulation, the mid-point of the transmission link could be accurately defined. 


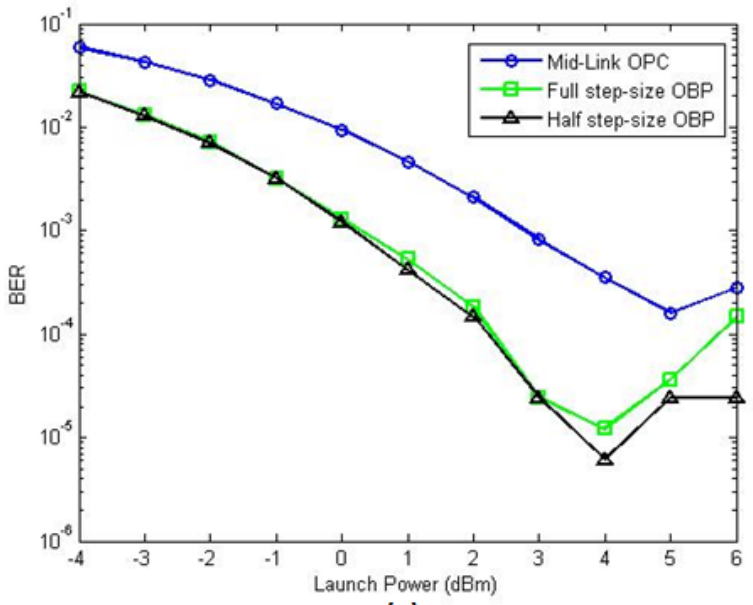

(a)

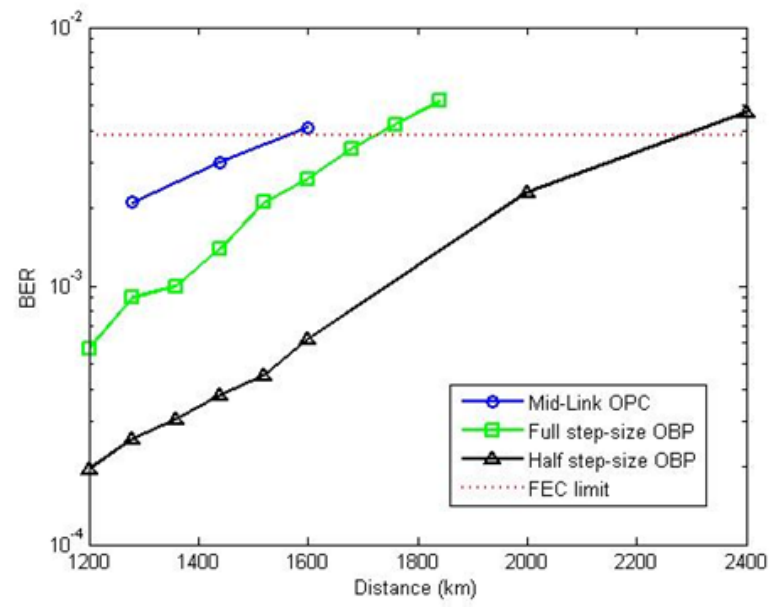

(b)

Fig. 2.6 Ideal OBP \& OPC performance, (a) BER v.s. launch power and (b) transmission reach test 


\begin{tabular}{|c|c|c|c|}
\hline Launch Power & Mid-Link OPC & FSS OBP & HSS OBP \\
\hline$-1 d B m$ & 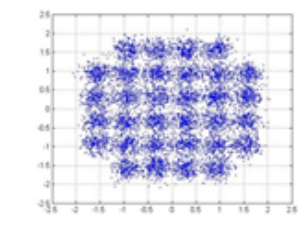 & 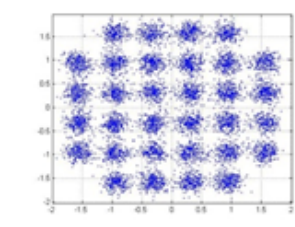 & 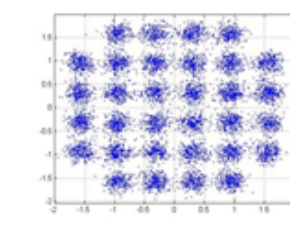 \\
\hline $2 d B m$ & 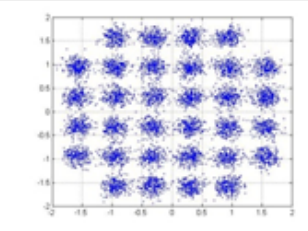 & 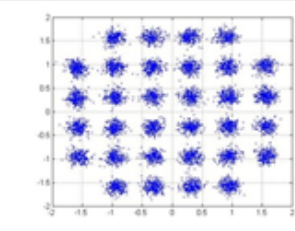 & 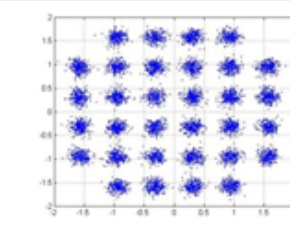 \\
\hline $5 d B m$ & 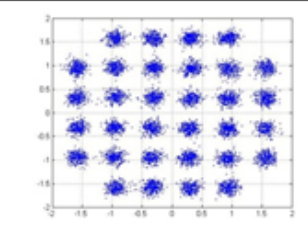 & 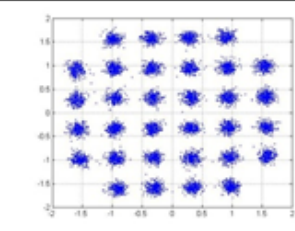 & 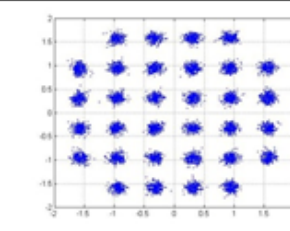 \\
\hline
\end{tabular}

Table 2.3 Constellation maps for different configurations under different launch power 


\section{Chapter 3}

\section{Simulation Results of OBP with Imperfection}

Previous measurements and comparisons in chapter 2 showed that the OBP method for nonlinearity control in optical fibers has a better performance compared with DBP and Midlink OPC. However, all the previous systems are based on ideal conditions assumptions, for instance, the OPC process has no degradation and all the optical in-line amplifiers have ideal noise figures, which is $3 d B$. Since there are solid proofs in section 1.2 that the optical phase conjugation process will introduce several up to dozens decibels of OSNR degradation in the system, it is necessary and useful to quantify the effects of this phenomenon. In this section, the influences of the OPC process caused OSNR degradation on the OBP systems performances are studied. The effects of the in-line amplifier noise figure on the systems are also discussed. Section 3.1 will simulate the influence of the OSNR degradation and section 3.2 will simulate the influence of in-line amplifiers noise figure.

\subsection{OBP Simulation with OSNR Degradation in OPC Process}

If we take the OSNR degradation of the OPC process into consideration, both the full stepsize and half step-size OBP performances will be affected. The experiment result in [28] suggests an at least $5 d B$ to more than $10 d B$ OSNR degradation in the system after the FWM wavelength conversion device. We gradually increase the degradation of OSNR after the OPC process from $0 d B$ to $5 d B$ by controlling the EDFA input into the $99: 1$ coupler and quantify the systems performances again. Figure 3.1 shows the result. 


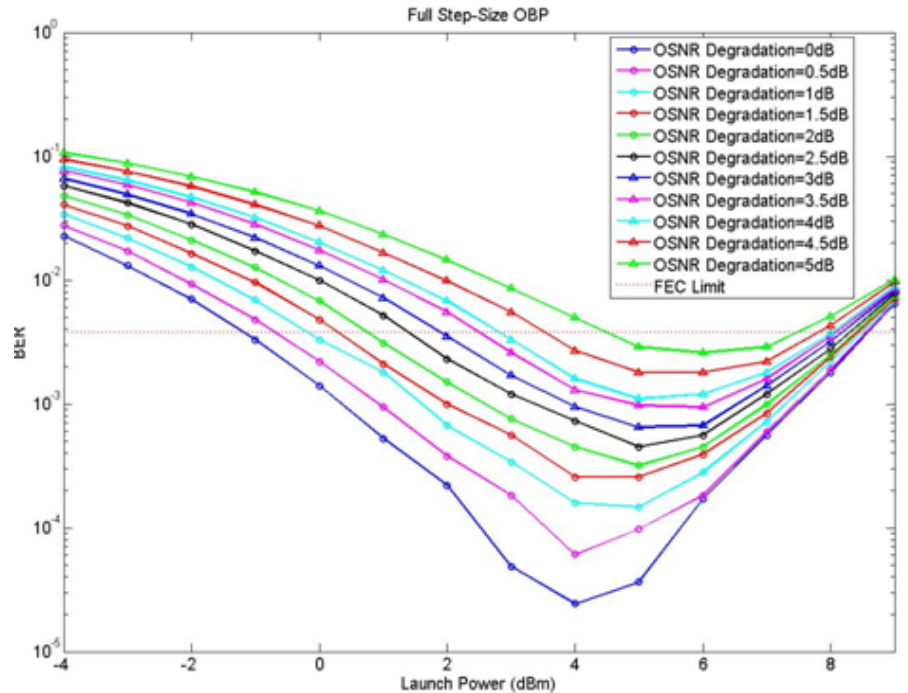

(a)

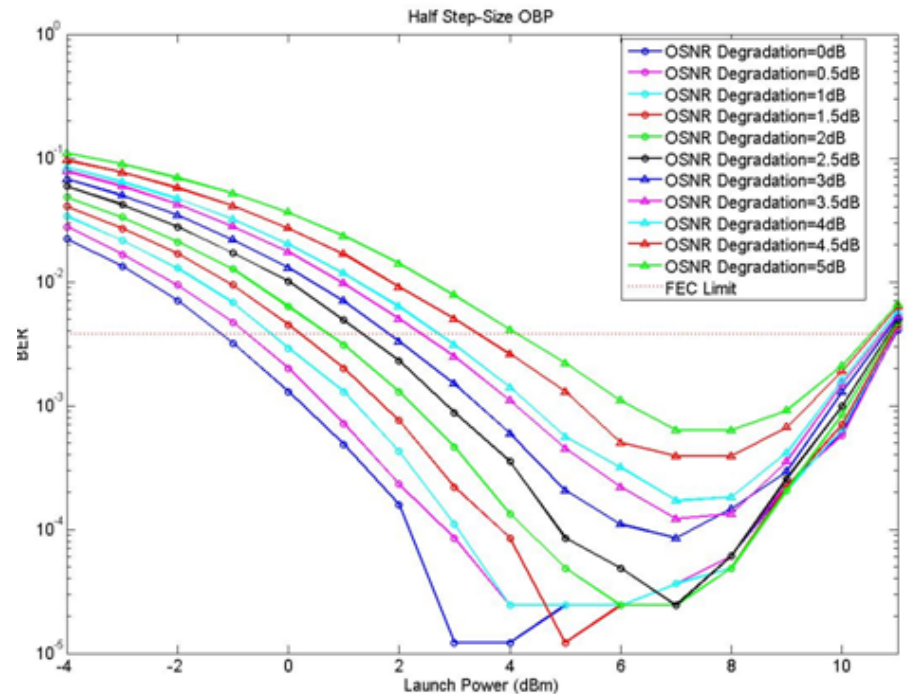

(b)

Fig. 3.1 OBP with OPC OSNR degradation measurements, (a) full step-size OBP and (b) half step-size OBP

Figure 3.1 (a) is the BER measurements versus launch power for full step-size OBP with an OSNR degradation in the system from $0 d B$ up to $5 d B$, (b) is the same measurement for half step-size OBP. The launch power is pushed to the limit that all the BER curves will cross the FEC limit twice for both measurements. With a maximum of $5 d B$ OSNR degradation after the phase conjugator, the optimized launch power pushes to a higher level and the BER degradation is obvious. It is clearly shown that the half step-size OBP 
performs better than full step-size OBP under the same circumstances. To have a clearer view of the degraded OSNR effect on the systems, the power penalty as a function of the OSNR degradation is also plotted. The power penalty is defined as the difference of launch power at which the system can just get error free recovery (when the bit error rate exactly equals the FEC limit) under different OSNR degradations. Since all the BER curves cross the FEC limit twice, the power penalty is also measured at both low power side and high power side.

The first two figures measured the power penalty of the full (blue curve)/half (green curve) step-size OBP at low (lower than $4 \mathrm{dBm}$ ) and high (higher than $8 \mathrm{dBm}$ ) power sides respectively versus the OSNR degradation in the system. At low system OSNR degradation (less than $4 d B$ ), the two step-size OBP show nearly the same power penalty at both launch power levels. When the systems OSNR degradation is higher than $4 d B$, the full step-size OBP starts to show a higher power penalty than the half step-size one, and the difference is more obvious at high launch power level. The last two figures qualified the optimized BER and launch power in relationship with the OSNR degradation. With a $5 d B$ of OSNR degradation, there could be a power penalty nearly $6 d B$ resulted in the system, which is high enough to take into consideration when doing other measurements or experiments in OBP systems. To complete the measurements on the system, the OSNR degradation effect on the signal to noise ratio (SNR) of the system is also studied. The SNR is measured at the systems output, it roughly follows the relationship between BER and SNR as the curves shown in Figure 3.3 (b). Figure 3.3 (a) shows the SNR of the system output signal as a function of the system launch power under different OSNR degradations, with a higher OSNR degradation, it is trivial that the output signal SNR would be lower.

The transmission reach distance test is also performed to test the longest distance the system can reach before the BER is higher than the FEC limit. Figure 3.4 shows the BER versus transmission distance measurements of the system measured under different OSNR degradations. It is trivial that with higher OSNR degradation in the system, the signal will travel shorter distance until it could not be recovered error free. It is also shown that, under the same OSNR degradation in the system, the signal in half step-size OBP can travel further than the full step-size one. In the full step-size OBP system, at the FEC limit of $3.8 \times 10^{-3}$, under ideal condition, the transmission reach limits to about $1550 \mathrm{~km}$ while with $5 d B$ of OSNR degradation during the OPC process, the transmission reach is limited to about $850 \mathrm{~km}$, which is almost only half of the transmission distance compared to the 


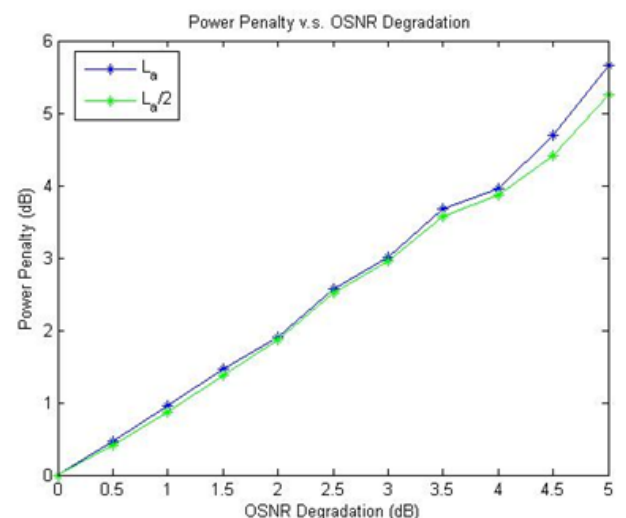

(a)

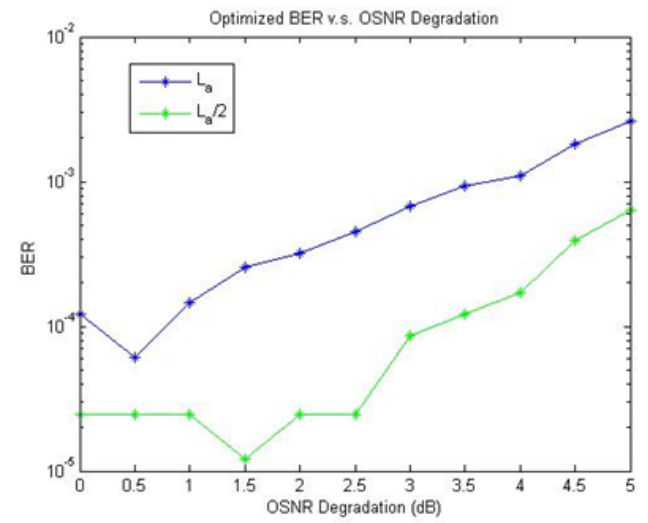

(c)

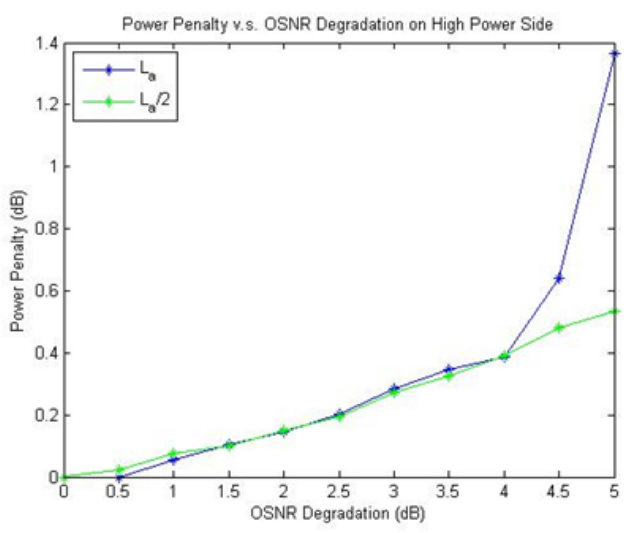

(b)

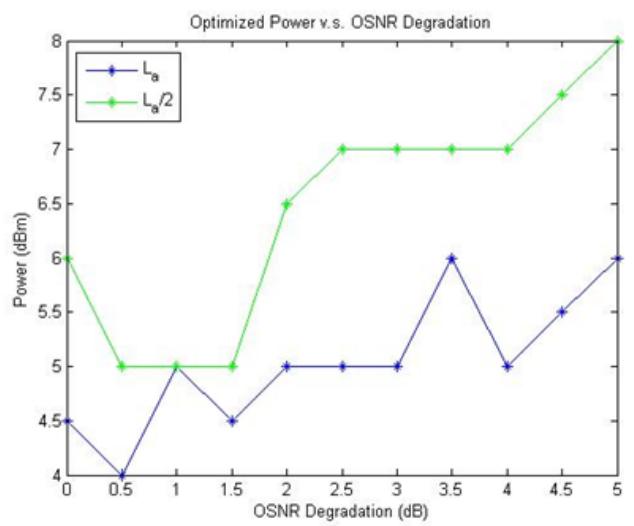

(d)

Fig. 3.2 Power penalty for OBP with imperfect OPC OSNR degradation \& optimized BER and launch power v.s. OSNR degradation, power penalty v.s. OSNR degradation at (a) low launch power and (b) high launch power. (c) Optimized BER v.s. OSNR degradation and (d) optimized system launch power v.s. OSNR degradation 


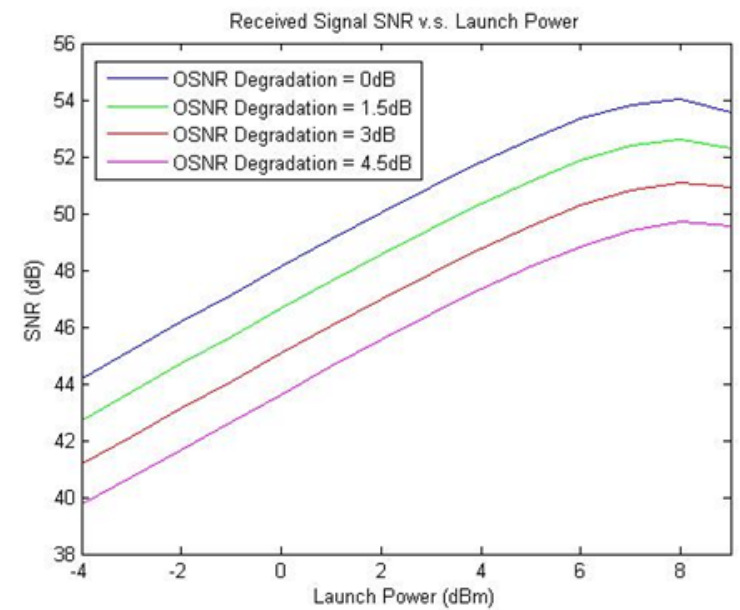

(a)

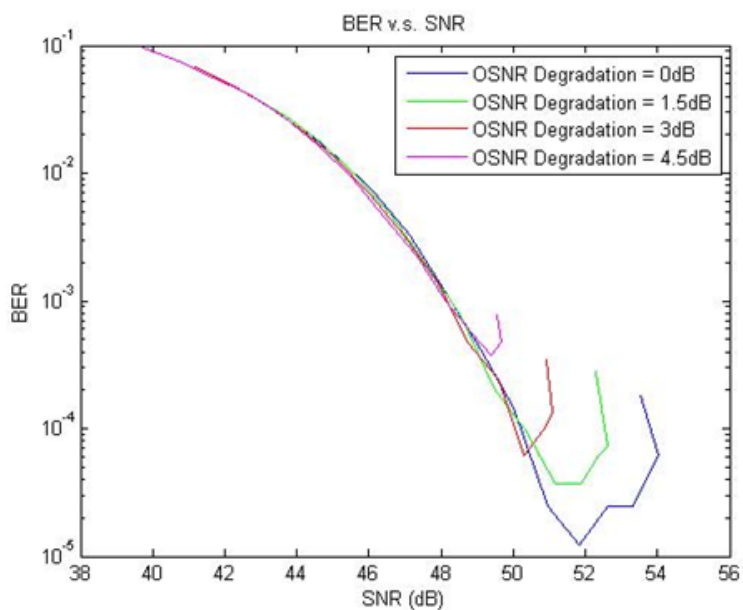

(b)

Fig. 3.3 (a) System output SNR performance on different OSNR degradation and (b) BER v.s. SNR relationship

ideal case. In the half step-size OBP system, the longest transmission distance under FEC limit is for the ideal condition with a distance of $2300 \mathrm{~km}$ while the shortest transmission distance is less than $1000 \mathrm{~km}$ when the OPC process introduces $5 \mathrm{~dB}$ of OSNR degradation to the system. That results in a more than half of length reduction of the transmission distance with error free recovery.

The percentage of the transmission distance penalty is also performed. From the data shown in Figure 3.5 we can see, with a $5 d B$ of OSNR degradation during the OPC process, the transmission distance of the signal in the system under FEC limit is reduced by nearly 60 percent for half step-size OBP and almost 50 percent for full step-size OBP. 


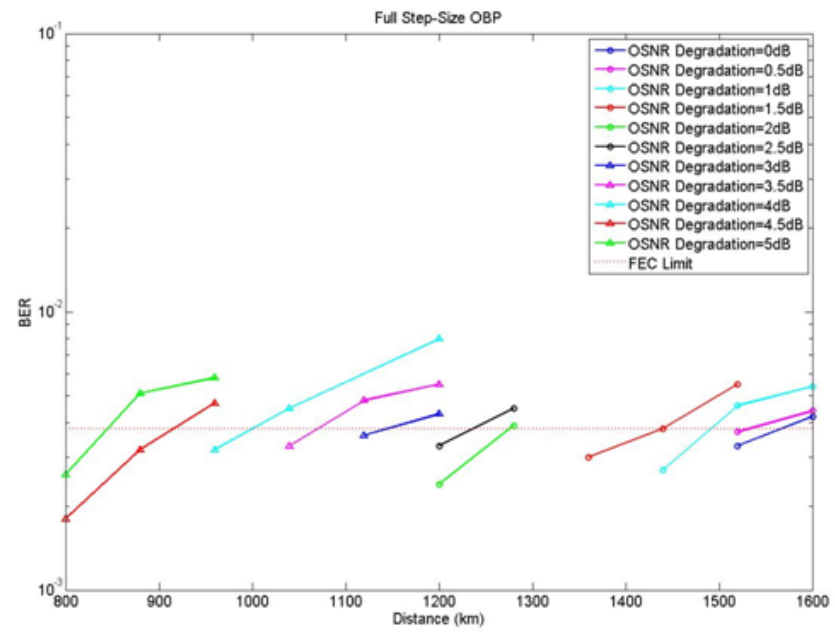

(a)

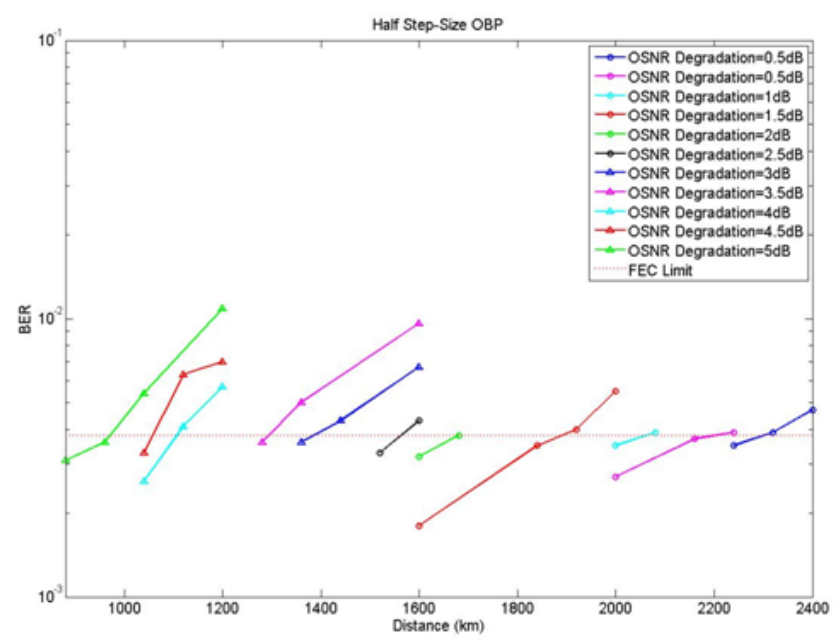

(b)

Fig. 3.4 Reach test for OBP with imperfect OPC OSNR degradation for (a) full step-size and (b) half step-size

\subsection{OBP Simulation with Different in-line Amplifier Noise Figure}

Besides the OPC process caused OSNR degradation in the system, the in-line amplifiers noise figure (NF) could also affect the performance of OBP. All the previous measurements are based on the ideal noise figure assumption for EDFAs $\left(n_{s p}=2\right)$, while in real case, a noise figure of $3 d B$ is really difficult to be achieved. In this section, the influence of the in-line amplifier noise figure on the OBP systems performances is studied. 


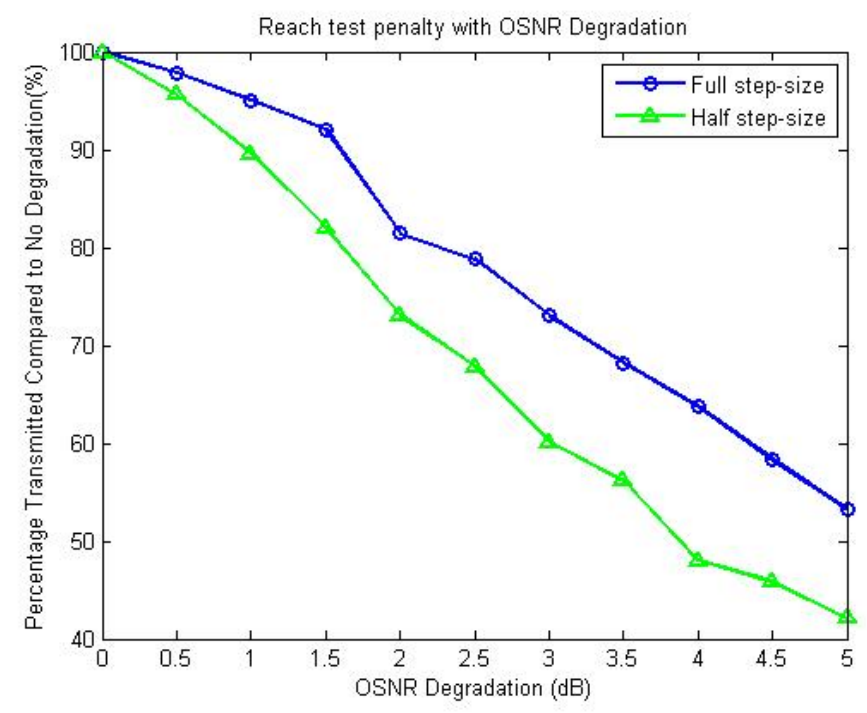

Fig. 3.5 Reach percentage under different OSNR degradation for FSS OBP \& HSS OBP

In this section, we compare the performances of the systems with the in-line amplifier noise figure of $4.5 \mathrm{~dB}$ and $12 \mathrm{~dB}$. The OPC process degraded the system with an OSNR degradation from $0 d B$ up to $4.5 d B$ by a step size of $1.5 d B$. We choose the values of $4.5 d B$ and $12 d B$ of noise figures because EDFAs can have a $\mathrm{NF}$ as low as $4.5 d B$ whereas SOAs can have a NF as high as $12 d B$. The results are shown in Figure 3.6.

The simulation results shown above indicate that, there is not much difference shown in the systems performances with different in-line amplifier noise figures. However in Figure 3.6 (a), we can still observe that, with the same amount of noise added by the amplifier to the system, the noise affects more on the system with lower OSNR degradation. It is reasonable to have this result since a clear system would more likely to be degraded by the additional amplified noise.

One of the reasons why the in-line amplifiers noise figure does not affect the system performance very much is that, there is an electrical amplifier in the coherent receiver and a band-pass filter. The electrical amplifier and the band-pass filter would also add noise to the system, the noise added by the in-line optical amplifier could be merged into this noise so that the overall effect is not obvious.

To summarize, this chapter simulated OBP systems under two non-ideal cases, following shows the results: 


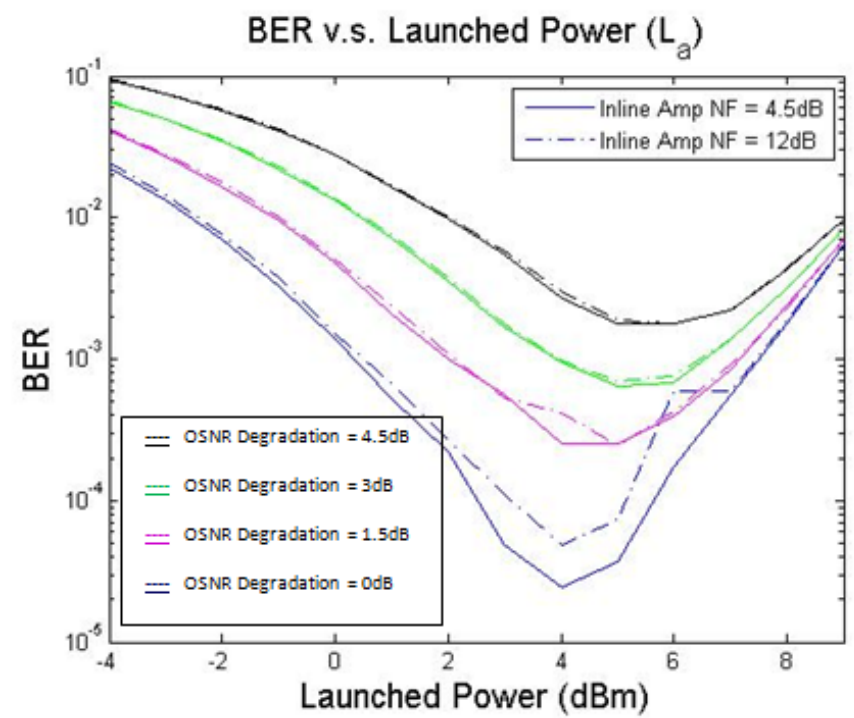

(a)

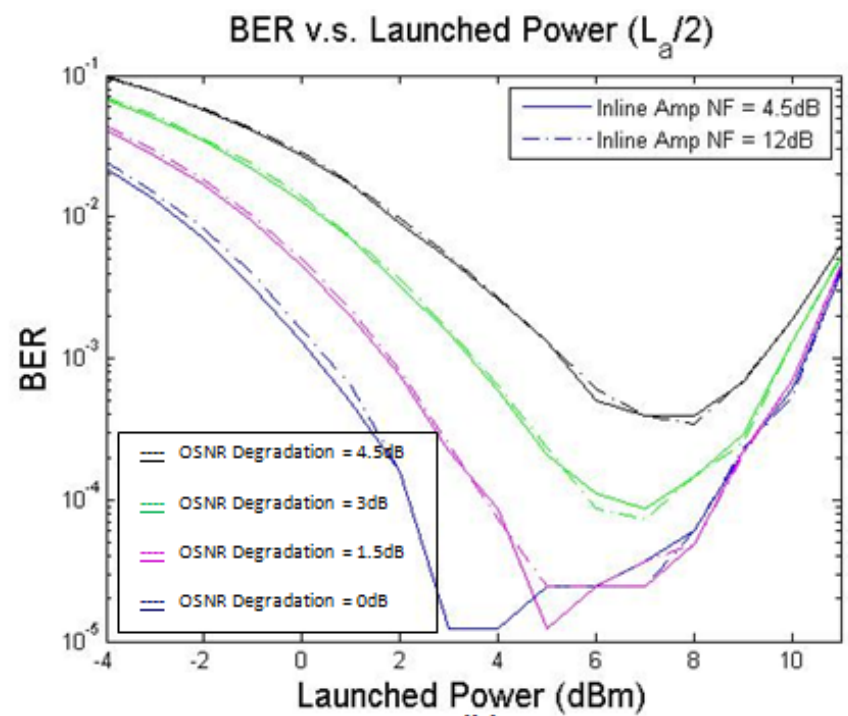

(b)

Fig. 3.6 System performances with in-line amplifier $N F=4.5 d B \& 12 d B$ for (a) full step-size OBP and (b) half step-size OBP

OBP with non-ideal $O P C$

An about $5 d B$ power penalty would occur when there is $5 d B$ of OSNR degradation 
introduced during the OPC process to the system. The transmission reach distance under FEC limit would also be reduced by about half. The optimised launch power to achieve the lowest BER of the systems with OSNR degradations would be higher than that with ideal conditions. It would need to re-adjust the systems launch power and take the power penalty into consideration when conducting OBP experiments in the lab.

\section{OBP with non-ideal in-line amplifier}

The normal EDFAs we used in the lab, without considering damages, would have a noise figure in the range of $4.5 d B$ to $6 d B$. While in this range of noise figure, the OBP systems will not experience very much degradation in the outputs. The in-line amplifier noise figure is not crucial to OBP systems. 


\section{Chapter 4}

\section{Simulation Results of OBP that Employs Different Baud Rate and Modulation Format}

In the previous chapter, the effects of non-ideal OPC process and in-line amplifier noise figures on the OBP systems have been discussed. The results showed that the OSNR degradation in the system would cause serious effects such as optimised launch power shift, error free recovery power penalty and error free recovery transmission distance shorten while the in-line amplifier noise figure does not affect the system too much. In this chapter, we further explore the OBP systems performances under different baud rates and different modulation formats. A reasonable prediction is that, under higher modulation formats and higher baud rates, the systems performance will be degraded to some extent, while the simulation results in this chapter will numerically verify those degradations on the systems performances. The effects of OSNR degradation and in-line amplifier noise figure on these systems will also be studied. Section 4.1 will discuss the effects of a higher baud rate on the systems and section 4.2 will discuss the effects of a higher modulation format.

\subsection{OBP Simulation with Different Baud Rate}

Increasing the signal baud rate in a system even by a slight number would have an obvious effect on the systems performance, especially when DWDM technique is employed. In this 
section, we compare the OBP systems performances with baud rates of $25 \mathrm{GBaud} / \mathrm{s}$ and $28 \mathrm{GBaud} / \mathrm{s}$. The $32 Q A M$ modulation format will be kept and all the in-line amplifiers have the same noise figure of $4.5 \mathrm{~dB}$. Furthermore, the OSNR degradation effects on the system will also be included.

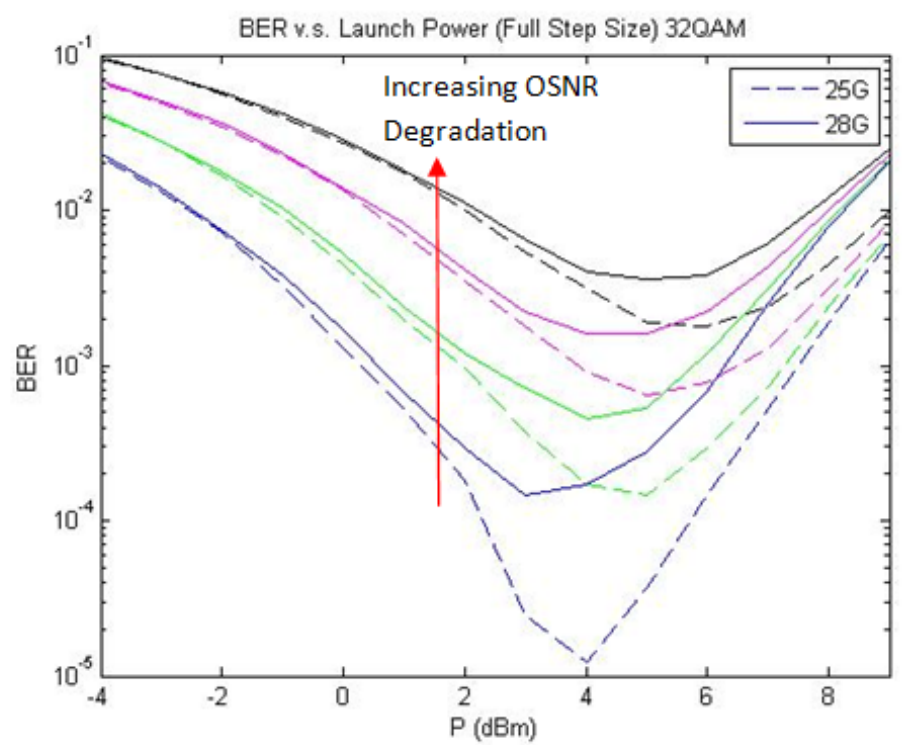

(a)

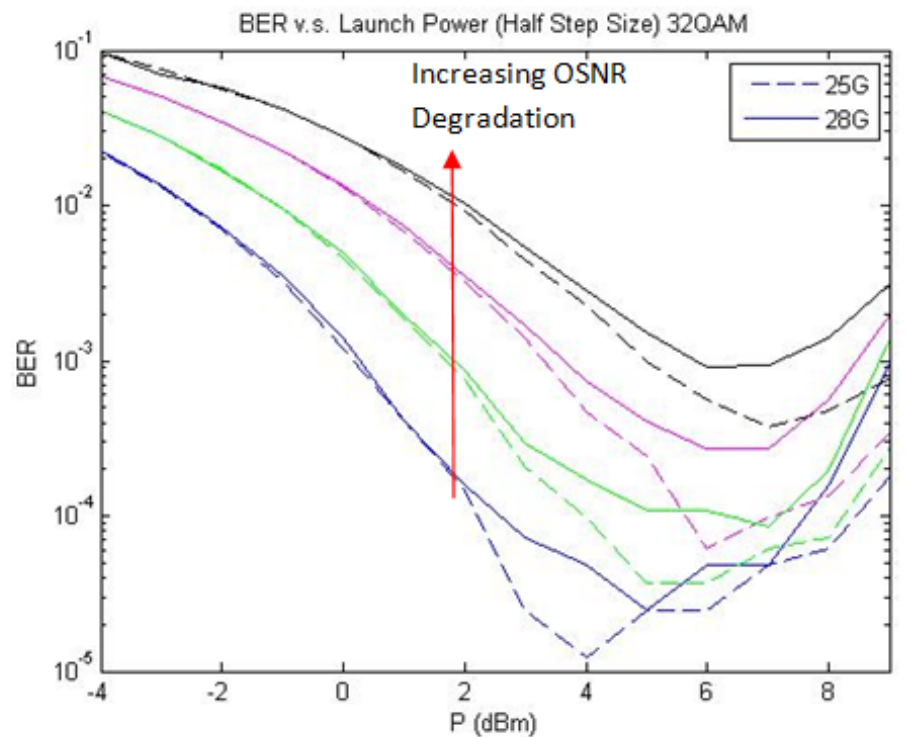

(b)

Fig. 4.1 System performances of OBP with 25 Gbaud/s \& 28 Gbaud/s for (a) full step-size and (b) half step-size 
Figure 4.1 shows the comparison of two systems with different baud rates. The signal baud rate at low launch power $(-4 \mathrm{dBm}$ to $2 \mathrm{dBm})$ does not affect the system outputs bit error rate significantly and it starts to become critical when the launch power is higher than $2 \mathrm{dBm}$. Comparing the two OBP schemes, the power penalty due to the baud rate change for full step-size system is higher than that for half step-size system. Moreover, the optimised launch power (when the system output has the lowest BER) is pushing to a lower level when the signal baud rate increases. There is an about $1 d B$ power penalty for full stepsize OBP with no OSNR degradation in the system to reach a bit error rate of FEC limit when the signal baud rate changes from $25 G$ to $28 G$ and the power penalty for half stepsize OBP under same conditions is about $0.5 \mathrm{~dB}$. The results in chapter 3 showed that the in-line amplifier noise figure does not have significant effects on the systems performances. With a higher baud rate, the systems performances are not comparable as that at the lower baud rate, it is reasonable to predict that the in-line amplifier noise figure would have even less effect on the $28 \mathrm{GBaud} / \mathrm{s}$ systems than the $25 \mathrm{GBaud} / \mathrm{s}$ ones. The comparison results for full/half step size OBP 28GBaud systems performances with in-line amplifier noise figures of $4.5 d B$ and $12 d B$ are shown in Figure 4.2 .

With the numerical analysis above, the result indeed does not show very much difference with respect to different in-line amplifier noise figures. With the in-line amplifier noise figure of $12 \mathrm{~dB}$, the BER of the system is just slightly higher than that of the system with a noise figure of $4.5 d B$. The effect of the noise figure change on the systems BER performances is higher when the OSNR degradation in the system is lower which agrees with the results in chapter 3 .

\subsection{OBP Simulation with Different Modulation Format}

Previous result in section 4.1 showed an about $1 \mathrm{~dB}$ power penalty when the system baud rate changes from $25 G$ Baud to $28 G$ Baud for a full step-size OBP system output to reach the FEC limit and $0.5 d B$ for half step-size OBP system. In this section, the effects of different modulation formats on the systems will be studied. To compare the results, $25 G$ baud rate applies to both cases, the simulations are for $32 Q A M$ and $64 Q A M$ modulation formats respectively. The in-line amplifiers noise figures are kept to be the normal value of $4.5 d B$.

The dashed curves in Figure 4.3 are for the same 25GBaud $32 Q A M$ signals used in 


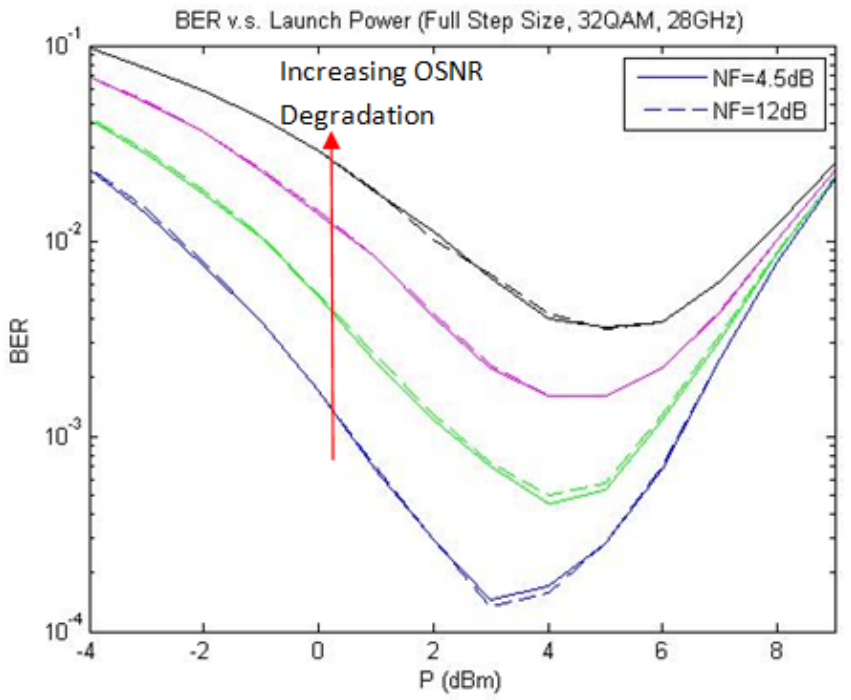

(a)

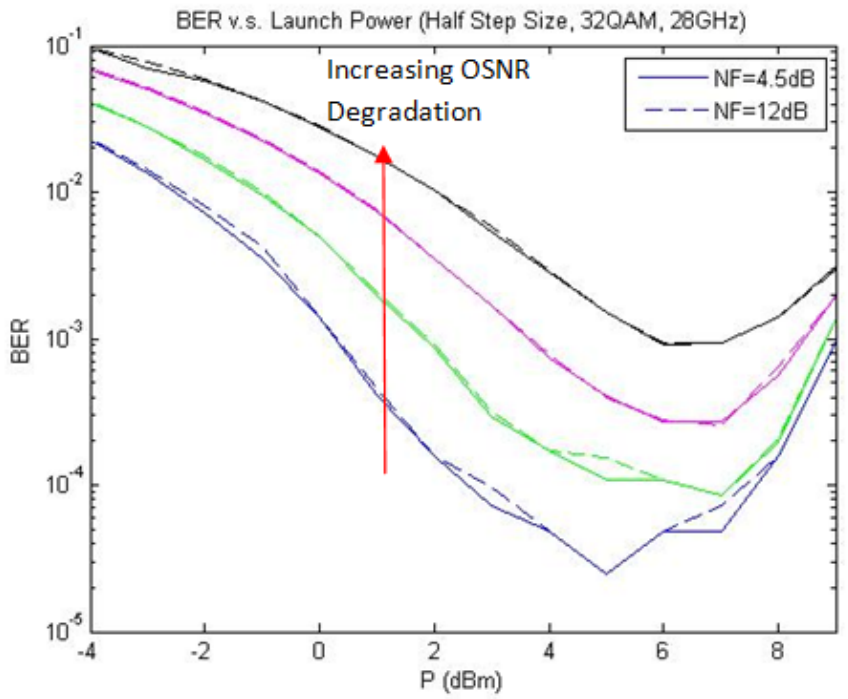

(b)

Fig. 4.2 System performances of OBP with $28 \mathrm{Gbaud} / \mathrm{s}$ for in-line amplifier noise figure of $4.5 d B$ and $12 d B$ for (a) full and (b) half step-size OBP

section 4.1. With the 64QAM modulation format, the BER of the system output is degraded significantly, even at low launch power. The optimised launch power to the system is pushed to a lower level with a higher modulation format. There is nearly a $4 d B$ of power penalty for full step-size OBP with no OSNR degradation to reach a bit error rate of $10^{-2}$ when change the $32 Q A M$ signal to $64 Q A M$ and the power penalty for half step-size OBP 


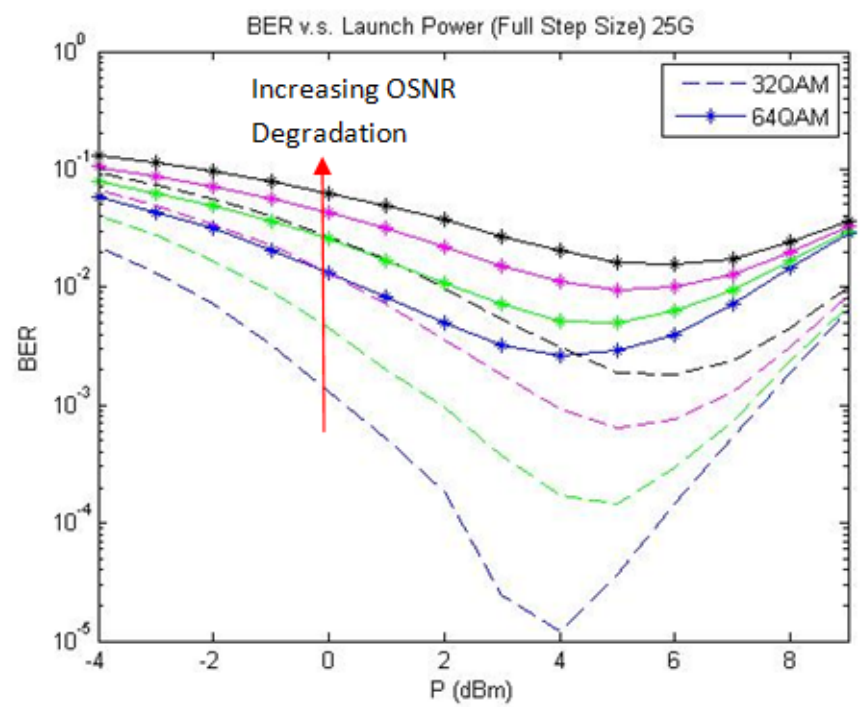

(a)

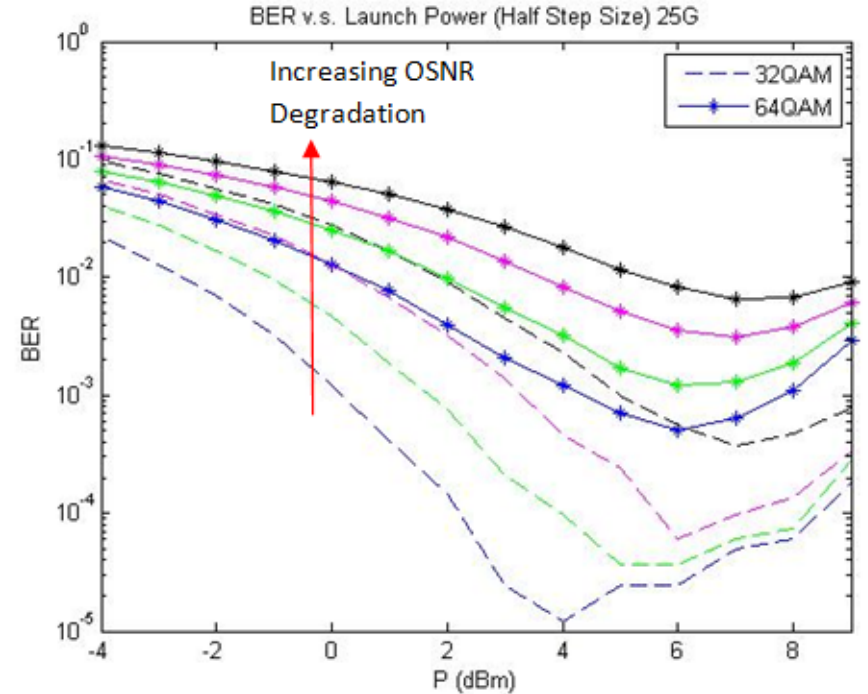

(b)

Fig. 4.3 System performances of OBP with $32 Q A M \& 64 Q A M$ modulated signals for (a) full step-size and (b) half step-size

under same conditions is also over $3 d B$. The power penalty becomes even higher when there is OSNR degradation presence in the system. Nearly all the BER curves will not cross the FEC limit when 64QAM modulation format adopted. To have a view of the constellation maps of the two modulation formats and compare the results, Table 4.1 is included below.

All the above constellation maps are for ideal systems, no OSNR degradation during 


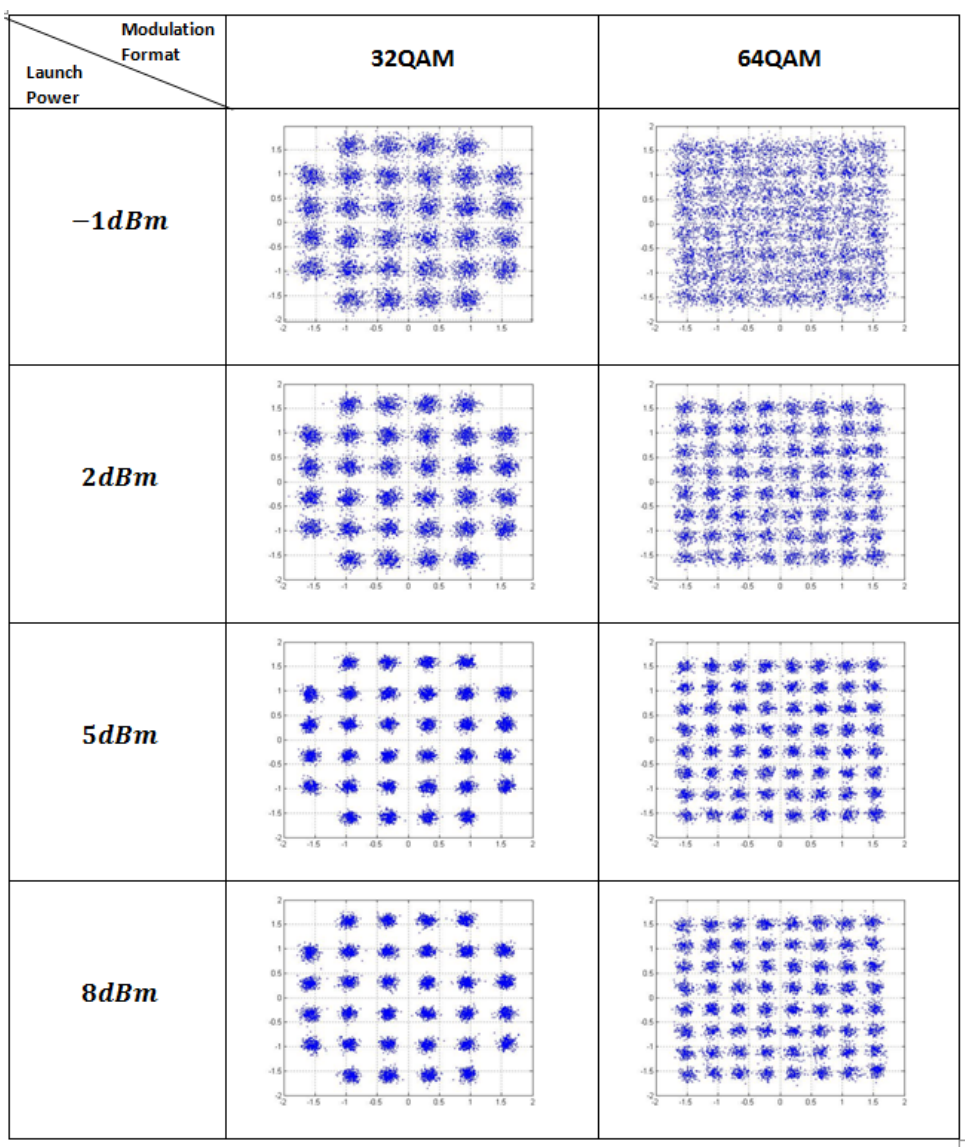

Table 4.1 Constellation maps for half step-size OBP with 25GBaud under different launch powers for $32 Q A M$ \& $64 Q A M$ 
the OPC process and the noise figure for in-line amplifiers are $4.5 d B$. To have a view of the effects of in-line amplifier noise figure on the $64 Q A M$ systems, the simulations of the system with different in-line amplifier noise figures are also performed.

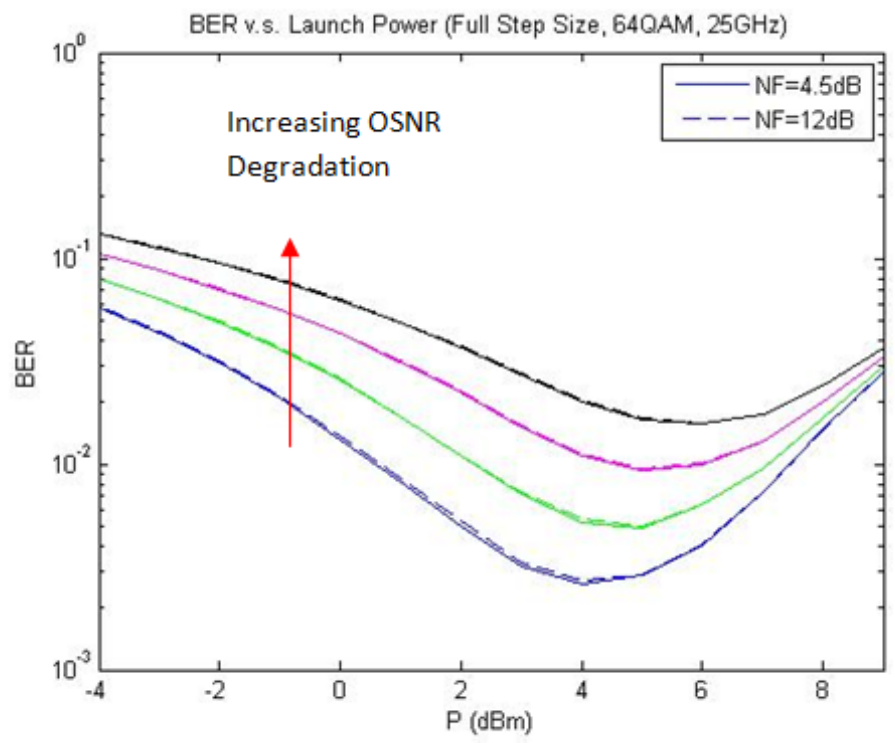

(a)

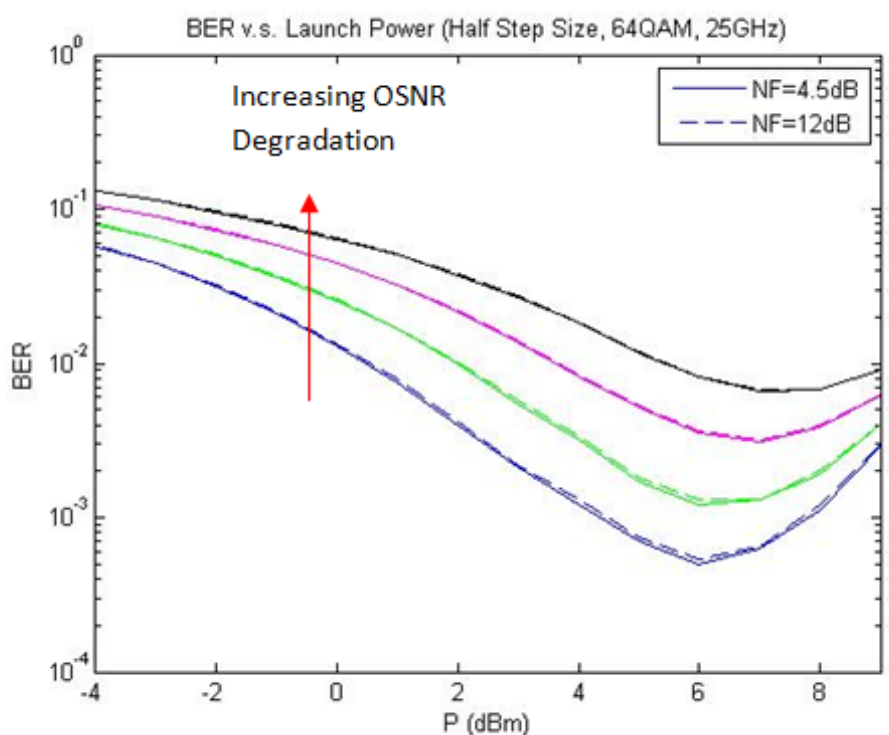

(b)

Fig. 4.4 System performances of OBP with in-line amplifier noise figures of $4.5 d B \& 12 d B$ for $64 Q A M$ modulated signals for (a) full and (b) half step-size

Figure 4.4 shows the performances of $64 Q A M$ system with in-line amplifier noise figures 
of $4.5 \mathrm{~dB}$ and $12 \mathrm{~dB}$. The results show no exceptions that the noise figures of EDFAs are not crucial in the system and they have more effects on clearer systems when no OSNR degradation appears.

With the simulations of OBP systems employ different baud rates and modulation formats, the analyses have been completed. To summarize the simulation results:

\section{OBP with different baud rates and modulation formats}

It is trivial that, in general systems, a higher signal baud rate or a higher modulation format will result in a degraded system performance. Our simulation shows that when the signal baud rate is higher or the modulation format is higher, the optimised launch power is lower, which should be taken into consideration when doing further experiments. 


\section{Chapter 5}

\section{Conclusion \& Future Work}

\subsection{Conclusion}

Internet, multi-media communications and services that brought to users by optical fibers are indispensable in everyday life in this information explosion age. The development of economy and technology has a result of uninterruptedly increasing of users that enjoy the services carried by optical fiber cables [38]. The increasing number of users and data requires large transmission capacity in fiber optic communication systems. In todays long haul fiber optic communication systems, the major problem that draws back the increasing of transmission capacity is the fiber nonlinearity. However it is not always necessary to add transmission capacity by building new lines, techniques such as optical back propagation has been established for nonlinearity control in optical fibers can also help. Although the technique of OBP has been established for some time, all the simulations done up to date are based on ideal systems assumptions. There are several ineluctable imperfections in the system that is necessary to take into account, such as the imperfection in the OPC process and the imperfection of the EDFAs. These imperfections are highly expected to happen and are useful to be characterized.

This thesis qualified the effects of the imperfections in OBP systems and analysed the results. It explored the influence of OSNR degradation which caused by optical phase conjugation on OBP systems. It also investigated the influence of EDFAs noise figure changes on the systems. Comparisons between different baud rates and modulation formats are also discussed.

In this thesis, chapter 1 illustrated the eager of increasing transmission capacity in 
fiber optic communication systems and discussed its major hurdle fiber nonlinearity. In this chapter, several nonlinear control methods, such as Mid-Link OPC, DBP and OBP, were introduced and the associated advantages and disadvantages were discussed. The motivation for optical back propagation was given.

Chapter 2 gave a brief introduction of the receiver based optical back propagation technique and discussed its developments and advantages. It showed the literature review of the OBP technique and justified some simulation results. The detailed working principles and parameter settings for OBP systems were also given. The overview of the necessary building blocks for OBP simulation was also given in this chapter.

Chapter 3 talked about the effects of some imperfections on the OBP systems. Given that there are demonstrations that several decibels of OSNR degradation would happen after the optical phase conjugation, this chapter characterized the effects of this degradation on the OBP systems. The results showed that the launch power needs to be re-adjusted in order to suffer less power penalty in the system. The effects of the in-line amplifier noise figures on the systems were also characterised, while the results showed that the systems were quite robust to the in-line amplifiers noise figures changes.

Chapter 4 simulated the OBP systems with $28 G$ baud rate and $64 Q A M$ modulation format. The results showed that, when higher baud rate or higher modulation format employed, the optimised launch power to the system should be lower. The effects of OSNR degradation and EDFA noise figure changing were also simulated on these systems.

\subsection{Future Work}

The work demonstrated in this thesis is focused on the effects of imperfections on receiver based OBP systems performances. All the works are simulations done by combine using MATLAB and OptiSystem. These results can also be used to determine the optimum operating system parameters under non-ideal conditions.

With this work demonstrated the working principles and performances of receiver based OBP, other optical back propagation schemes can also be developed sharing similar working principles. For instance, the OBP spans at the receiver can be separated and preferably located into each amplifier span of the transmission link. Then in each of the transmission span, the dispersion and nonlinear effects could be compensated immediately before they become accumulated more. Since the OBP sets are located in the amplifier spans, there is no 
need to define any mid-point of the transmission link. The in-line OBPs working principle is identical to the receiver based one but employs more OPC sets in the system. Since the complexity of the system is higher for in-line OBP, it could be a good prospectiveness if the BER performance or the FEC limit reach distance of the system significantly outperforms the receiver based one.

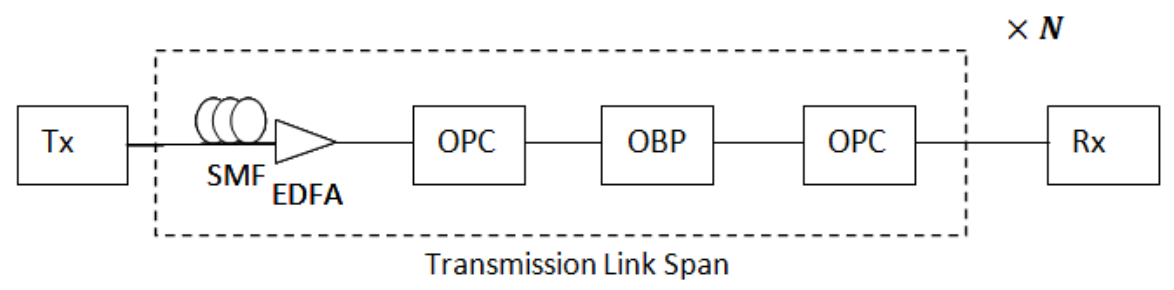

Fig. 5.1 In-line OBP schematic diagram

A similar way of doing in-line OBP is to preferably locate the HNLFs in the transmission span while with the chromatic dispersion compensating at the receiver, optically (using HDFs or DCFs or FBGs) or electrically (using DSP). The accumulated chromatic dispersion in the transmission spans will broaden the pulse spectrum and make the channel more "quasi-linear" like, so that the nonlinear effect would not build up significantly.
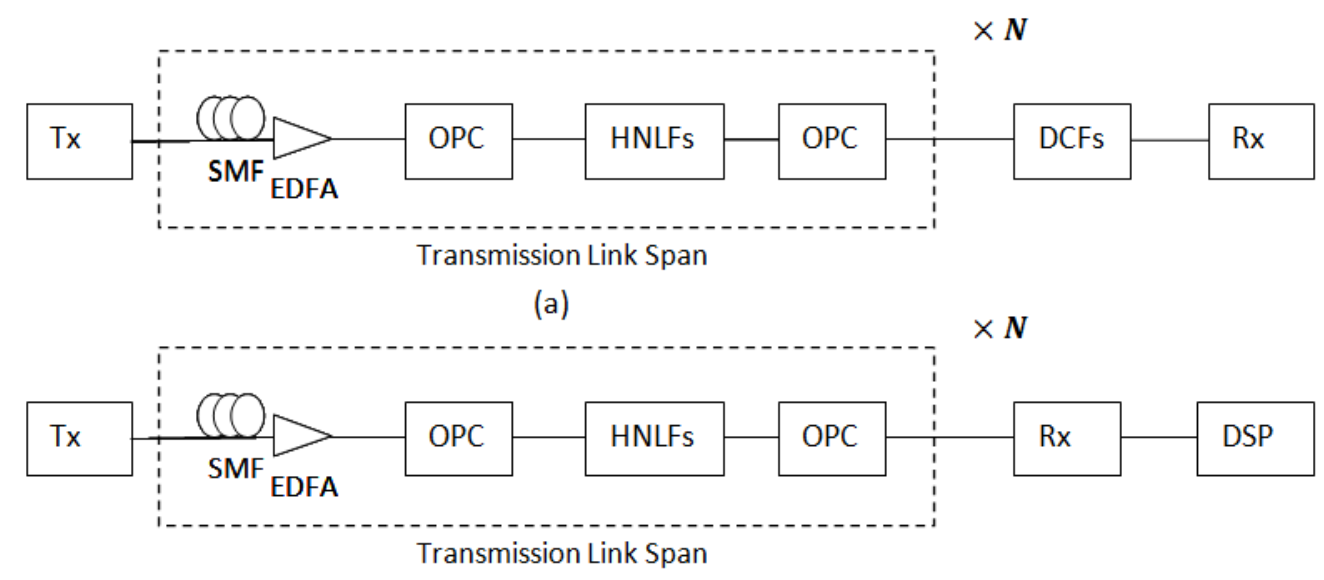

(b)

Fig. 5.2 In-line nonlinear compensation with CD compensate (a)optically and (b)electrically 
Another perspective of this work is to test the systems tolerance of the parameters of HDFs and HNLFs. All the above measurements are based on the accurately calculated parameters, to test the tolerance of the systems, we could add some variance to the parameters (such as the dispersion coefficient $D$ of HDFs and the nonlinear coefficient $\gamma$ of the HNLFs) in each span. The idea is shown in the following figure.

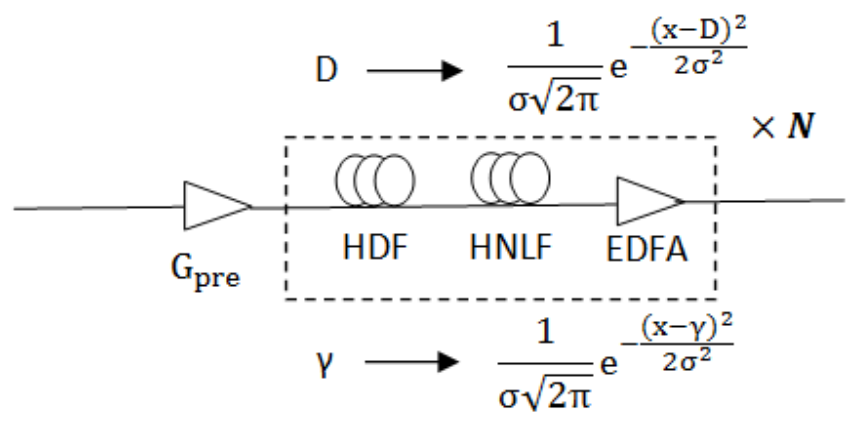

Fig. 5.3 OBP span parameters with variation

This work in this thesis is based on mathematical simulations, the next step can take to experimental investigations and verifications of the simulation results. Other performance qualification factors such as $Q$-factor or eye-diagram can also be included in the extension of this work. 


\section{References}

[1] M. A. Shampo, R. A. Kyle, and D. P. Steensma, "Charles K. Kao - Father of Fiber Optics," in Mayo Clinic Proceedings, vol. 86, p. e45, Mayo Foundation, 2011.

[2] V. Alwayn, "Fiber-Optic Technologies," Retrieved March, vol. 12, p. 2012, 2004.

[3] D. Richardson, J. Fini, and L. Nelson, "Space-division multiplexing in optical fibres," Nature Photonics, vol. 7, no. 5, pp. 354-362, 2013.

[4] G. P. Agrawal, Fiber-optic communication systems, vol. 1. 1997.

[5] P. J. Winzer, "Modulation and multiplexing in optical communications," in Conference on Lasers and Electro-Optics, p. CTuL3, Optical Society of America, 2009.

[6] P. J. Winzer and R. Essiambre, "Advanced optical modulation formats," Proceedings of the IEEE, vol. 94, no. 5, pp. 952-985, 2006.

[7] W. C. Lee, "Estimate of channel capacity in rayleigh fading environment," Vehicular Technology, IEEE Transactions on, vol. 39, no. 3, pp. 187-189, 1990.

[8] P. P. Mitra and J. B. Stark, "Nonlinear limits to the information capacity of optical fibre communications," Nature, vol. 411, no. 6841, pp. 1027-1030, 2001.

[9] G. Agrawal, Applications of nonlinear fiber optics. Academic press, 2001.

[10] A. R. Chraplyvy et al., "Limitations on lightwave communications imposed by opticalfiber nonlinearities," Journal of Lightwave Technology, vol. 8, no. 10, pp. 1548-1557, 1990.

[11] S. P. Singh and N. Singh, "Nonlinear effects in optical fibers: Origin, management and applications," Progress In Electromagnetics Research, vol. 73, pp. 249-275, 2007.

[12] G. P. Agrawal, Lightwave technology: telecommunication systems. John Wiley \& Sons, 2005. 
[13] J. Shao and S. Kumar, "Optical backpropagation for fiber-optic communications using optical phase conjugation at the receiver," Optics letters, vol. 37, no. 15, pp. 30123014, 2012.

[14] R. Asif, C.-Y. Lin, and B. Schmauss, "Digital backward propagation: a technique to compensate fiber dispersion and non-linear impairments," InTech-Book Publisher, 2011.

[15] E. Ip and J. M. Kahn, "Compensation of dispersion and nonlinear impairments using digital backpropagation," Lightwave Technology, Journal of, vol. 26, no. 20, pp. 34163425, 2008.

[16] D. Rafique, J. Zhao, and A. D. Ellis, "Fundamental limitations of digital backpropagation in coherent transmission systems," in Transparent Optical Networks (ICTON), 2011 13th International Conference on, pp. 1-4, IEEE, 2011.

[17] C. Janer and M. Connelly, "Optical phase conjugation technique using four-wave mixing in semiconductor optical amplifier," Electronics letters, vol. 47, no. 12, pp. 716-717, 2011 .

[18] R. Yu, N. K. Fontaine, R. Proietti, B. Guan, and S. Yoo, "All-Optical Phase Conjugation Using a 90 degree Optical Hybrid and Nested SOA-MZIs," in Optical Fiber Communication Conference, pp. OW4C-6, Optical Society of America, 2013.

[19] S. Jansen, D. van den Borne, P. Krummrich, G. Khoe, and H. de Waardt, "Experimental Comparison of Optical Phase Conjugation and DCF Aided DWDM 2x10. 7Gbit/s DQPSK Transmission," Citeseer.

[20] S. Kumar and D. Yang, "Optical backpropagation for fiber-optic communications using highly nonlinear fibers," Optics letters, vol. 36, no. 7, pp. 1038-1040, 2011.

[21] X. Liang, S. Kumar, and J. Shao, "Ideal optical backpropagation of scalar NLSE using dispersion-decreasing fibers for wdm transmission," Optics Express, vol. 21, no. 23, pp. 28668-28675, 2013.

[22] M. Ducloy, "Nonlinear optical phase conjugation," in Festkörperprobleme 22, pp. 3560, Springer, 1982.

[23] D. M. Pepper, "Nonlinear optical phase conjugation," Optical Engineering, vol. 21, no. 2, pp. 212156-212156, 1982.

[24] M. Jang, A. Sentenac, and C. Yang, "Optical phase conjugation (OPC)-assisted isotropic focusing," Optics express, vol. 21, no. 7, pp. 8781-8792, 2013. 
[25] T. T. Grove, E. Rousseau, X.-W. Xia, D. Hsiung, M. Shahriar, and P. Hemmer, "Efficient and fast optical phase conjugation by use of two-photon-induced gratings in the orientation of angular momentum," Optics letters, vol. 22, no. 22, pp. 1677-1679, 1997.

[26] S. Watanabe, S. Kaneko, and T. Chikama, "Long-haul fiber transmission using optical phase conjugation," Optical Fiber Technology, vol. 2, no. 2, pp. 169-178, 1996.

[27] T. R. Hillman, T. Yamauchi, W. Choi, R. R. Dasari, M. S. Feld, Y. Park, and Z. Yaqoob, "Digital optical phase conjugation for delivering two-dimensional images through turbid media," Scientific reports, vol. 3, 2013.

[28] R. Adams, M. Spasojevic, M. Chagnon, M. Malekiha, J. Li, D. Plant, and L. Chen, "Four Wave Mixing Based Wavelength Conversion and Multicasting of 16-QAM Signals in a Silicon Nanowire," in Photonics Conference (IPC), 2013 IEEE, pp. 1-2, IEEE, 2013.

[29] R. Adams, M. Spasojevic, M. Chagnon, M. Malekiha, J. Li, D. V. Plant, and L. R. Chen, "Wavelength conversion of 28 GBaud 16-QAM signals based on four-wave mixing in a silicon nanowire," Optics express, vol. 22, no. 4, pp. 4083-4090, 2014.

[30] G. P. Agrawal, Nonlinear fiber optics. Springer, 2000.

[31] M. Malekiha, D. Yang, and S. Kumar, "Comparison of optical back propagation schemes for fiber-optic communications," Optical Fiber Technology, vol. 19, no. 1, pp. 4-9, 2013.

[32] J. Y. Leong, P. Petropoulos, J. H. Price, H. Ebendorff-Heidepriem, S. Asimakis, R. C. Moore, K. E. Frampton, V. Finazzi, X. Feng, T. M. Monro, et al., "High-nonlinearity dispersion-shifted lead-silicate holey fibers for efficient $1-\mu \mathrm{m}$ pumped supercontinuum generation," Lightwave Technology, Journal of, vol. 24, no. 1, pp. 183-190, 2006.

[33] X. Li, X. Chen, G. Goldfarb, E. Mateo, I. Kim, F. Yaman, and G. Li, "Electronic post-compensation of WDM transmission impairments using coherent detection and digital signal processing," Optics Express, vol. 16, no. 2, pp. 880-888, 2008.

[34] D. Rafique and A. D. Ellis, "Nonlinearity compensation via spectral inversion and digital back-propagation: A practical approach," in Optical Fiber Communication Conference, pp. OM3A-1, Optical Society of America, 2012.

[35] P. Minzioni, F. Alberti, and A. Schiffini, "Techniques for nonlinearity cancellation into embedded links by optical phase conjugation," Lightwave Technology, Journal of, vol. 23, no. 8, pp. 2364-2370, 2005. 
[36] S. Jansen, D. Van Den Borne, P. Krummrich, S. Spalter, G. Khoe, and H. De Waardt, "Long-haul DWDM transmission systems employing optical phase conjugation," $S e$ lected Topics in Quantum Electronics, IEEE Journal of, vol. 12, no. 4, pp. 505-520, 2006.

[37] M. D. Pelusi, "WDM signal all-optical precompensation of Kerr nonlinearity in dispersion-managed fibers," Photonics Technology Letters, IEEE, vol. 25, no. 1, pp. 71$74,2013$.

[38] V. Alwayn, Optical network design and implementation. Cisco Press, 2004. 